\title{
The structure of optimal statin initiation policies for patients with Type 2 diabetes
}

\author{
MURAT KURT $^{1}$, BRIAN T. DENTON ${ }^{2, *}$, ANDREW J. SCHAEFER ${ }^{1}$, NILAY D. SHAH $^{3}$ \\ and STEVEN A. SMITH ${ }^{4}$ \\ ${ }^{1}$ Department of Industrial Engineering, University of Pittsburgh, Pittsburgh, PA 15261, \\ E-mail:muk7@pitt.edu,E-mail: schaefer@ie.pitt.edu \\ ${ }^{2}$ Edward P. Fitts Department of Industrial and Systems Engineering, North Carolina State University, Raleigh, NC 27695, \\ E-mail: bdenton@ncsu.edu \\ ${ }^{3}$ Divisions of Health Care Policy and Research, and Knowledge and Encounter Research Unit, Mayo Clinic College of Medicine, \\ Rochester, MN 55905, \\ E-mail: shah.nilay@mayo.edu \\ ${ }^{4}$ Divisions of Endocrinology, and Health Care Policy and Research, Mayo Clinic College of Medicine, Rochester, MN 55905, \\ E-mail: smith.steven@mayo.edu
}

Received September 2010 and accepted December 2010

\begin{abstract}
Lipid abnormalities increase the risk of coronary heart disease (CHD) and stroke in patients with Type 2 diabetes. Statins can be used to treat these abnormalities, but may have adverse side effects. In this article, we consider the optimal timing of statin initiation for patients with Type 2 diabetes. We formulate an infinite-horizon Markov decision process to maximize the patient's quality-adjusted life years (QALYs) prior to the occurrence of the first CHD or stroke event. We describe the state of the process by the patient's lipid ratio levels, and derive structural properties of the resulting optimal stopping time model, including sufficient conditions for the optimality of control-limit policies with respect to patient's lipid ratio levels and age. We use a large clinical data set to parameterize our model and compute optimal treatment policies to demonstrate the clinical implications of our results. We illustrate the importance of individualized treatment factors by estimating the patients' QALY gains using our customized policies rather than current U.S. guidelines.
\end{abstract}

Keywords: diabetes, Markov decision processes, control-limit policy

\section{Introduction}

There are currently 20.8 million Americans with diabetes (American Diabetes Association (ADA) 2008), and this number is expected to grow to 39 million by 2050 (Honeycutt et al., 2003). Type 2 diabetes is caused by insulin resistance combined with relative insulin deficiency and accounts for the $90 \%$ of the diabetes cases in the U.S. Diabetes patients often have difficulty maintaining their blood glucose levels within healthy ranges. Historically, the primary goal of managing Type 2 diabetes has been the control of blood glucose levels; however, more recently, the importance of cardiovascular risk has been emphasized (The United Kingdom Prospective Diabetes Study (UKPDS) Group 1998, Snow et al. 2004).

\footnotetext{
${ }^{*}$ Corresponding author
}

Clinical trials have shown that cholesterol management using statins reduces CHD and stroke risks (Downs et al. 1998, Collins et al. 2002; 2003, Colhoun et al. 2004, Briel et al. 2004). The complexity of these treatment decisions has led to the development of several national treatment guidelines. One of the published U.S. guidelines, known as Adult Treatment Panel (ATP) III, sets cholesterol targets based on patient's 10-year CHD risk (National Cholesterol Education Program (NCEP) 2001). ATP III guidelines were modified to treat the presence of diabetes an explicit cardiovascular risk factor, resulting in tighter cholesterol targets (see Appendix C for details). Moreover, some guidelines (Snow et al. 2004, ADA 2008) recommend initiating statins in all patients with Type 2 diabetes irrespective of their long-term CHD risks.

Although statins reduce the risk of CHD and stroke, they can have serious side effects, including muscle diseases and liver problems. Other less significant effects, such as headaches, nausea, fever, and fatigue, are more common 
(Phillips et al., 2002, Pasternak et al., 2002). However, none of the published guidelines explicitly consider the trade-off between the benefits of reduction in CHD and stroke risks and the adverse side effects of treatment.

Quality-adjusted life years (QALYs) are commonly used for treatment evaluation and health policy investigations (Gold et al., 2002). We use QALYs to adjust raw life years to account for the reduction in quality of life associated with side effects from statin treatment and consider the question of if and when to initiate statin treatment to maximize a Type 2 diabetes patient's QALYs prior to the occurrence of the first CHD or stroke event, or death from all other causes. We refer to these three outcomes collectively as terminal events.

We formulate the statin initiation problem as a discretetime infinite-horizon Markov decision process (MDP). We derive analytical results about the structure of the optimal policy, and present empirical results based on a large longitudinal data set from Mayo Clinic in Rochester, MN. The specific contributions of our work are as follows. First, we develop a model that can guide physicians and patients regarding the initiation of statins. Second, we derive sufficient conditions for the optimal policy to exhibit control-limits, and characterize how such limits change with respect to patient's age. Our results support the structure of existing treatment guidelines. Third, we present numerical results based on a large longitudinal data set to empirically confirm our analytical results and provide insights into the effect of side effects of statins on treatment decisions. Finally, we compare published U.S. guidelines to the optimal policies generated by our model.

To our knowledge, ours is the first optimization model that provides individualized recommendations for the optimal timing of statin initiation, demonstrating the influence of various CHD and stroke risk factors on the patient's optimal treatment policy. Denton et al. (2009) is the only study we are aware of that models the optimal timing of statin initiation. In contrast to this article, which focuses on the patient's perspective and side effects of treatment, they model the problem from a societal perspective. Our work differs from Denton et al. (2009) also in that we provide analytical evidence for the optimality of intuitively appealing treatment policies.

The remainder of this paper is organized as follows: In Section 2 we describe our MDP model. In Section 3 we analyze the structure of the resulting optimal policies of our model, which we support by a series of numerical experiments in Section 4. In Section 5 we present conclusions and summarize the limitations of our work.

\section{Model formulation}

We formulate a discrete-time, infinite-horizon MDP model for the optimal timing of statin initiation for patients with Type 2 diabetes. Once a patient with Type 2 diabetes initiates statin treatment, clinical guidelines recommend that she should continue for treatment in the remainder of her life (Vijan and Hayward 2004, Snow et al., 2004). We treat statin initiation as a one-time irreversible decision. Specifically, the decision we consider is if and when to initiate statin treatment over the course of the patient's lifetime based on her risk profile to maximize her QALYs prior to the first terminal event.

There are several published risk models that predict the CHD and stroke probabilities for patients with Type 2 diabetes based on their cholesterol levels and other risk factors. The most popular of these models was calibrated on data from the UKPDS study (Turner 1998, Stevens et al., 2001, Kothari et al., 2002). The UKPDS model predicts CHD and stroke probabilities based on several risk factors, including age, gender, ethnicity, smoking status, cholesterol, systolic blood pressure (SBP) and hemoglobin A1c (HbAlc) levels. While other predictive models have been developed (e.g. the Framingham model (Anderson et al., 1991) and the Archimedes model (Eddy and Schlessinger 2003a, 2003b)), the UKPDS model is unique in that it has published risk equations that are specific to patients with Type 2 diabetes.

Following the UKPDS risk model, we describe the patient's CHD and stroke risk profile by her cholesterol, SBP and $\mathrm{HbA} 1 \mathrm{c}$ levels, and her age, gender, ethnicity and smoking status at diagnosis. Of these risk factors, the patient's age, cholesterol, SBP and HbA1c levels are dynamic. Based on clinical evidence that patient's SBP and HbA1c levels are correlated with age, the studies that use stochastic models to analyze the cost-utility effects of therapies for diabetes treatment assume time-dependent SBP and HbA1c levels (Burt et al., 1995, Kannel 1996, Kilpatrick et al., 1996, Carrera et al., 1998, Hajjar and Kotchen, 2003). Similar to these studies, we incorporate the effect of the patient's age into her CHD and stroke risk profile by a time index and model the evolution of the patient's SBP and HbAlc levels deterministically as a function of time.

Elevated total cholesterol (TC) and depressed highdensity lipoprotein (HDL), also referred to as "good" cholesterol, have been reported to increase the overall risk of CHD and stroke. The ratio of TC to HDL, defined as the lipid ratio (LR), is a commonly used predictor of CHD and stroke risks, in models such as UKPDS (Wannamethee et al., 2000, Vijan and Hayward 2004). However, this ratio can vary significantly and unpredictably over time (Garber et al., 1994). Therefore, we model the progression of the patient's LR levels as a finite-state discrete-time Markov chain. We describe the components of our MDP model as follows:

Time horizon: We assume the treatment decision is revisited periodically (e.g. annual visits to an endocrinologist) over the patient's lifetime prior to the occurrence of the first terminal event. We define $T=\{0,1,2, \ldots, N\}$ as the set of decision epochs and assume the problem parameters at epoch $N-1$ remain stationary beyond epoch $N-1$. 
Therefore, the epochs beyond $N-1$ are not differentiated and are represented by $N$. This modeling approach reflects the nonstationary nature of risk with respect to age, and provides an approximation framework for the patient's remaining expected QALYs prior to the first terminal event over the time horizon for which there is insufficient medical data due to low sample sizes for high ages. For simplicity, we let $T^{\prime}=T \backslash\{N\}$ refer to the set of epochs in the nonstationary horizon, and we let stage $t$ refer to time interval between epochs $t$ and $t+1$. We assume the time between two successive decision epochs is $\tau>0$ years.

States: We discretize the continuous range of LR values into a finite set of states $\mathcal{L}^{\prime}=\{1,2, \ldots, L\}$ where each $\ell \in$ $\mathcal{L}^{\prime}$ corresponds to an $L R$-range and lower indexed ranges indicate lower values of LR. We add an absorbing state $L+1$ to the set of LR-ranges to denote a terminal event, and let $\mathcal{L}=\mathcal{L}^{\prime} \cup\{L+1\}$. Note that our state description also involves static risk factors, including age at diagnosis, gender, smoking status, and dynamic (but non-stochastic) risk factors: SBP, HbA1C. Because these factors are not affected by the patient's treatment status, for notational convenience we suppress the dependency on them.

Actions: The action in state $\ell \in \mathcal{L}$ is chosen from $\{W, I\}$, where $W$ represents waiting for one more stage and $I$ stands for initiating treatment immediately. We assume that using statins reduces the patient's LR level by a factor $\omega$ which we call the patient's treatment-effect factor. We define the treatment status of the patient by a binary indicator $m \in \mathcal{M}=\{0,1\}$, where " 0 " and " 1 " refer to not using and using statins, respectively. Because statin initiation is a onetime irreversible decision, once the treatment is initiated $m$ switches from 0 to 1 and remains as such.

Probabilities: We have four types of probabilities in our model: The probabilities of non-CHD or non-strokerelated death, CHD, stroke, and the transition probabilities among the LR-ranges. At epoch $t \in T^{\prime}$, a non-CHD or non-stroke-related death occurs with probability $d_{t}$. Otherwise, if the patient is in state $\ell \in \mathcal{L}^{\prime}$ under treatment status $m \in \mathcal{M}$, a CHD event occurs with probability $\pi_{t}^{C}(\ell, m)$ and a stroke event occurs with probability $\pi_{t}^{S}(\ell, m)$. We let $\pi_{t}^{\nabla}(\ell, m)=\pi_{t}^{C}(\ell, m)+\pi_{t}^{S}(\ell, m)$ denote the patient's probability of having a CHD or a stroke event in state $\ell \in \mathcal{L}^{\prime}$ under treatment status $m \in \mathcal{M}$ in stage $t$.

Given the patient is in state $\ell \in \mathcal{L}$ under treatment status $m \in \mathcal{M}$ at epoch $t \in T^{\prime}$, the probability of moving into the absorbing state $L+1$ at epoch $t+1$ is denoted by $p_{t}^{m}(L+1 \mid \ell)$, where $p_{t}^{m}(L+1 \mid \ell)=d_{t}+\left[1-d_{t}\right] \pi_{t}^{\nabla}(\ell, m)$ for $(\ell, m) \in \mathcal{L}^{\prime} \times \mathcal{M}$ and $p_{t}^{m}(L+1 \mid L+1)=1$ for both $m \in \mathcal{M}$. Given the patient is in state $\ell \in \mathcal{L}^{\prime}$ at epoch $t \in T^{\prime}$ and does not incur a terminal event in stage $t$, the probability of being in state $\ell^{\prime} \in \mathcal{L}^{\prime}$ at the next epoch is denoted by $q\left(\ell^{\prime} \mid \ell\right)$. We define $p_{t}^{m}\left(\ell^{\prime} \mid \ell\right)$ to be the patient's probability of being in state $\ell^{\prime} \in \mathcal{L}$ at epoch $t+1$ given she is in state $\ell \in \mathcal{L}$ under treatment status $m \in \mathcal{M}$ at epoch $t \in T^{\prime}$, which we write as:

$$
p_{t}^{m}\left(\ell^{\prime} \mid \ell\right)= \begin{cases}{\left[1-p_{t}^{m}(L+1 \mid \ell)\right] q\left(\ell^{\prime} \mid \ell\right)} & \text { if } \ell, \ell^{\prime} \in \mathcal{L}^{\prime}, \\ p_{t}^{m}(L+1 \mid \ell) & \text { if } \ell \in \mathcal{L}^{\prime}, \ell^{\prime}=L+1, \\ 1 & \text { if } \ell=\ell^{\prime}=L+1, \\ 0 & \text { otherwise. }\end{cases}
$$

We let $\mathbf{P}^{m}(t)=\left[p_{t}^{m}\left(\ell^{\prime} \mid \ell\right)\right]$ denote the patient's transition probability matrix under treatment status $m \in \mathcal{M}$ at epoch $t \in T^{\prime}$.

Rewards: We define $r^{m}(\ell)=\tau(1-m \sigma)$ to be the immediate reward in QALYs accrued in state $\ell \in \mathcal{L}^{\prime}$ under treatment status $m \in \mathcal{M}$, where $\sigma \in[0,1]$ denote the quality-adjustment factor due to negative side effects of using statins (Pignone et al., 2006, Timbie et al., 2010). Since our objective is to maximize the patient's QALYs prior to the first terminal event, we set all the immediate rewards associated with the absorbing state $L+1$ to zero, i.e., $r^{m}(L+1)=0$ for both $m \in \mathcal{M}$. Figure 1 illustrates the states, transitions, and rewards, for a particular stage $t$. The single node on the left represents the patient's current LRrange at epoch $t$. The nodes on the right are partitioned into states for LR-ranges and terminal events.

In our model, the patient continues to accumulate rewards prior to his/her first CHD or stroke event. Once she incurs a terminal event she transitions into state $L+1$, which is absorbing and provides no rewards. Therefore, upon occurrence of a terminal event, the accumulation of rewards stops and the patient terminates the process. Since statin initiation is a one-time decision, we define $\mu_{t}(\ell)$ as the patient's expected post-treatment reward if the treatment is initiated in state $\ell \in \mathcal{L}$ at epoch $t \in T$, which can be recursively written as:

$$
\mu_{t}(\ell)= \begin{cases}r^{1}(\ell)+\lambda \sum_{\ell^{\prime} \in \mathcal{L}} p_{t}^{1}\left(\ell^{\prime} \mid \ell\right) \mu_{t+1}\left(\ell^{\prime}\right) & \text { for } \ell \in \mathcal{L} \text { and } t \in T^{\prime}, \\ r^{1}(\ell)+\lambda \sum_{\ell^{\prime} \in \mathcal{L}} p_{t-1}^{1}\left(\ell^{\prime} \mid \ell\right) \mu_{t}\left(\ell^{\prime}\right) & \text { for } \ell \in \mathcal{L} \text { and } t=N\end{cases}
$$

where $\lambda \in(0,1)$ is the discount factor per stage (Gold et al., 1996, Drummond et al., 1997). The expected posttreatment rewards can be viewed as parameters of the model since they are determined by a separate Markov reward chain. For a patient with no history of CHD or stroke in state $\ell \in \mathcal{L}$ at epoch $t \in T$, we let $u_{t}(\ell)$ denote the maximum total expected discounted QALYs prior to the first terminal event and we define $a_{t}(\ell)$ as the corresponding optimal action, which we determine by solving the following optimality equations:

$$
u_{t}(\ell)=\left\{\begin{array}{r}
\max \left\{r^{0}(\ell)+\lambda \sum_{\ell^{\prime} \in \mathcal{L}} p_{t}^{0}\left(\ell^{\prime} \mid \ell\right) u_{t+1}\left(\ell^{\prime}\right), \mu_{t}(\ell)\right\} \\
\text { for } \ell \in \mathcal{L} \text { and } t \in T^{\prime}, \\
\max \left\{r^{0}(\ell)+\lambda \sum_{\ell^{\prime} \in \mathcal{L}} p_{t-1}^{0}\left(\ell^{\prime} \mid \ell\right) u_{t}\left(\ell^{\prime}\right), \mu_{t}(\ell)\right\} \\
\text { for } \ell \in \mathcal{L} \text { and } t=N .
\end{array}\right.
$$




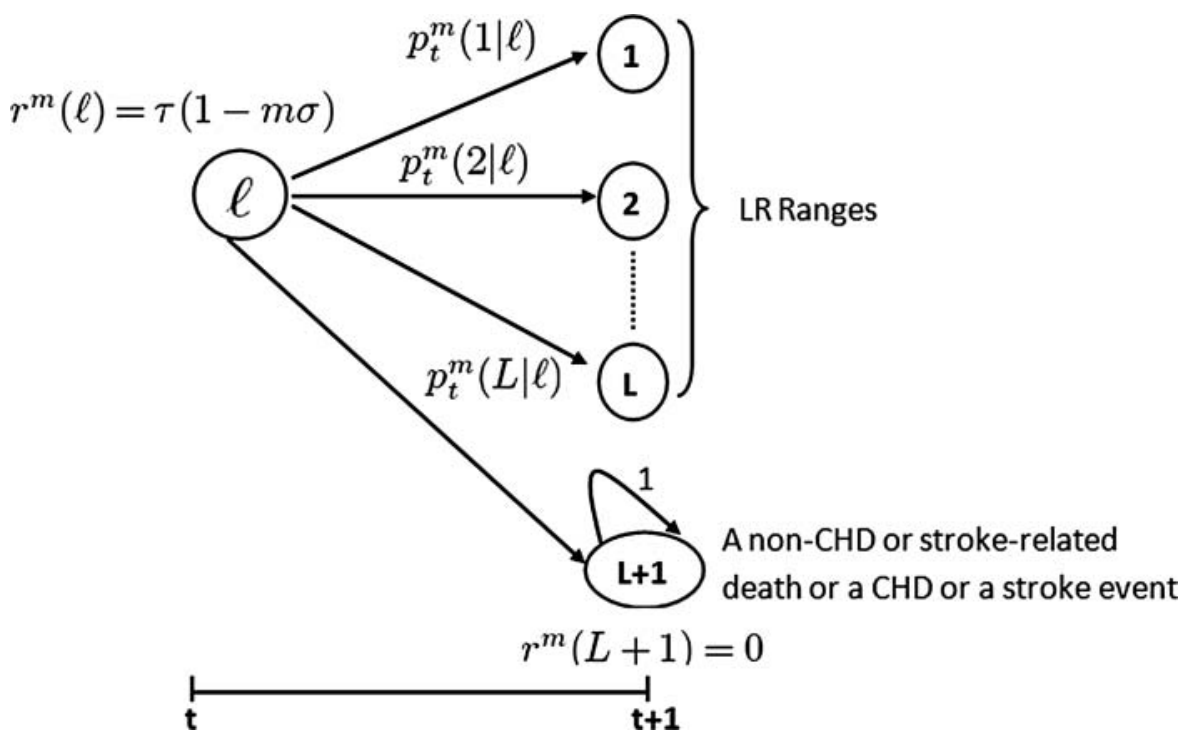

Fig. 1. State transition diagram at epoch $t \in T^{\prime}$ under treatment status $m \in \mathcal{M}$.

\section{Structural properties}

In this section we derive our main theoretical results. While MDP models have been applied in other clinical contexts (see Alagoz et al. (2010) for a review of discrete-time MDP models in health care), we provide several extensions. Specifically, Alagoz et al. (2004) provides sufficient conditions to prove an optimal control-limit policy within the context of living-donor liver transplantation. The authors consider a stationary infinite-horizon MDP. Although reasonable for liver transplant decisions this assumption is not appropriate for long-term chronic diseases, such as diabetes and heart disease, for which the risk of an event changes significantly with age. We provide less restrictive and more intuitive conditions to establish the optimality of controllimit policies in a non-stationary context, and analyze how the resulting control-limits change with respect to age (see Appendix D for the proofs of our results). The latter results are particularly noteworthy since most U.S. guidelines do not consider age as a risk-factor for the treatment of patients with diabetes.

The monotonic behavior of the optimal value function is commonly used to prove a threshold structure of the optimal policy in MDPs. In contrast, we define an expected benefit loss function and guarantee the optimality of control-limit policies given the monotonicity of this function. Therefore, our conditions do not require any special structure for the optimal value function of the model, and in this sense are less-restrictive than those of Alagoz et al. (2004).

In our problem context, we define a control-limit policy as follows: A patient should initiate treatment if and only if her LR is above some threshold. Note that many published national lipid management guidelines, including well known U.S. guidelines, have a control-limit structure (NCEP 2001, National Heart Foundation of Australia 2001, New Zealand Guidelines Group 2003, The British
Cardiac Society 2005, Fourth Joint Task Force 2007). Almost all of these guidelines define their thresholds in terms of the patient's long-term risk for a major cardiovascular event. Of these, the British and New Zealand guidelines also use explicit LR thresholds for their treatment recommendations.

We begin our analyses by defining the notion of an "increasing failure rate" (IFR) transition probability matrix, which has been shown to be useful in proving the structural properties of MDPs in maintenance and reliability theory (Valdez-Flores and Feldman 1989) and appears to match clinical data closely in varying contexts (Alagoz et al., 2004, Kreke et al., 2008).

Definition 1. (Barlow and Proschan 1965) An $n \times n$ stochastic matrix $\mathbf{H}=[h(j \mid i)]$ is said to have the IFR property if $\sum_{j=1}^{k} h(j \mid i)$ is nonincreasing in i for all $k=1, \ldots, n$.

In our model, under treatment status $m \in \mathcal{M}$ at epoch $t \in T^{\prime}$, if the matrix $\mathbf{P}^{m}(t)$ is IFR, an intuitive explanation is as follows: The higher the LR the patient has at epoch $t$ under treatment status $m$, the more likely she is to have a higher LR or to incur a terminal event at epoch $t+1$. By Proposition 1, given the patient's on- and offtreatment transition probabilities have the IFR property at each epoch, the maximum expected QALYs prior to the first terminal event does not increase in LR.

Proposition 1. If $\mathbf{P}^{m}(t)$ is IFR for all $m \in \mathcal{M}$ and $t \in T^{\prime}$, then the optimal value function, $u_{t}(\ell)$, is nonincreasing in $\ell \in \mathcal{L}$ for all $t \in T$.

Next, we define an expected benefit loss function which we use to express our main results. We let $B_{t}(\ell)$ denote the patient's expected benefit loss (in QALYs) from delaying the initiation of treatment to the next epoch in LR-range $\ell \in \mathcal{L}^{\prime}$ of epoch $t \in T^{\prime}$. More explicitly, for $\ell \in \mathcal{L}^{\prime}$ and $t \in T^{\prime}$, we define $B_{t}(\ell)=\mu_{t}(\ell)-\lambda \sum_{\ell^{\prime} \in \mathcal{L}} p_{t}^{0}\left(\ell^{\prime} \mid \ell\right) \mu_{t+1}\left(\ell^{\prime}\right)$. Note that 
since $B_{t}(\ell)$ is a function of the expected post-treatment rewards, it is a composite model parameter. Proposition 2 provides sufficient conditions for the optimality of LRbased control-limit policies based on the comparison of the patient's expected benefit loss in different LR levels.

Proposition 2. Suppose $\mathbf{P}^{0}(t)$ is IFR for all $t \in T^{\prime}$ and $B_{t}(\ell)$ is nondecreasing in $\ell \in \mathcal{L}^{\prime}$ for all $t \in T^{\prime}$. Then, there exists an LR threshold $\ell_{t}^{*} \in \mathcal{L}^{\prime}$ for each $t \in T$ such that the optimal action in state $\ell$ at epoch $t$ is to initiate treatment if and only if $\ell \geq \ell_{t}^{*}$, i.e., $a_{t}(\ell)=I$ for all $\ell \geq \ell_{t}^{*}$, and $a_{t}(\ell)=W$ otherwise.

Next, we focus on how the optimal policy changes with respect to age. We assume the patient's non-CHD or nonstroke-related death probability, $d_{t}$, is nondecreasing in $t \in T^{\prime}$. Note that this assumption is consistent with published mortality data (National Center for Health Statistics (NCHS) 2005). It is also well established that age is a positive-risk factor for CHD and stroke. We state this assumption formally as follows:

$$
\begin{gathered}
p_{t+1}^{m}(L+1 \mid \ell) \geq p_{t}^{m}(L+1 \mid \ell) \text { for all } \ell \in \mathcal{L}, m \in \mathcal{M}, \\
t \in T^{\prime} \backslash\{N-1\} .
\end{gathered}
$$

Proposition 3 expresses the time-monotonicity of the patient's optimal value function. Specifically, it states that the patient's expected QALYs prior to the first terminal event do not increase as she ages.

Proposition 3. The optimal value function, $u_{t}(\ell)$, is nonincreasing in $t \in T$ for all $\ell \in \mathcal{L}$.

Next, we explore the structure of the optimal policy with respect to age. Proposition 4 states that if the patient's expected benefit loss from delaying the initiation of treatment to the next epoch does not decrease over time then the optimal threshold for treatment does not increase as she ages.

Proposition 4. If $B_{t}(\ell)$ is nondecreasing in $t \in T^{\prime}$ for all $\ell \in$ $\mathcal{L}^{\prime}$, then for any $\ell \in \mathcal{L}^{\prime}$ and $t \in T^{\prime}, a_{t}(\ell)=I$ implies $a_{t+1}(\ell)=$ I.

In the remainder of this section, we will explore the sensitivity of the patient's optimal value function and policy with respect to quality-adjustment and treatment-effect factors. Proposition 5 compares two patients who are identical except for their quality-adjustment and/or treatment-effect factors, and addresses the relationship between the optimal policy and patient's response to treatment. For Patient $i$, we specify the problem parameters and the value functions by a pre-superscript, $i=1,2$.

Proposition 5. Consider two patients who are identical except ${ }^{2} \sigma \geq{ }^{1} \sigma$ and/or ${ }^{1} \omega \geq{ }^{2} \omega$. Then, for any $\ell \in \mathcal{L}^{\prime}$ and $t \in T$, ${ }^{2} a_{t}(\ell)=I$ implies ${ }^{1} a_{t}(\ell)=I$.

In Proposition 5, if it is optimal to initiate treatment at a given LR level, it must be optimal to do so if the patient's disutility of using statins decreases, and/or the treatment effect factor increases.

Finally, Proposition 6 establishes the sensitivity of the patient's optimal value function with respect to simultaneous changes in quality-adjustment and treatment-effect factors. It explicitly states that the lower the quality-adjustment factor the patient has, the higher the benefit she gains as the treatment effect increases.

Proposition 6. Consider two patients who are identical except ${ }^{1} \omega \geq{ }^{2} \omega$. Let $\sigma_{1}$ and $\sigma_{2}$ be two quality-adjustment factors, with $\sigma_{1} \geq \sigma_{2}$, and let ${ }^{i, j} u_{t}(\ell)$ specify ${ }^{i} u_{t}(\ell)$ when the qualityadjustment factor is $\sigma_{j}$, for $\ell \in \mathcal{L}, t \in T$ and $i, j=1,2$. Then, ${ }^{1,2} u_{t}(\ell)-{ }^{1,1} u_{t}(\ell) \geq{ }^{2,2} u_{t}(\ell)-{ }^{2,1} u_{t}(\ell)$ for all $\ell \in \mathcal{L}$ and $t \in T$.

Note that as a side result provided by Proposition 6, the patient's optimal value function does not decrease as $\sigma$ decreases and/or $\omega$ increases.

\section{Numerical study}

In this section, we present numerical results to illustrate our model. We also investigate the sensitivity of the patients' optimal value functions and policies with respect to their quality-adjustment and treatment-effect factors. Finally, we evaluate and compare the three most common U.S. guidelines with respect to optimal policies from our model.

We define two patient profiles, one for each gender, for which we present the resulting optimal treatment policies and the maximum expected QALYs prior to the first terminal event. We describe the details on data sources and estimation of model parameters in Appendix B.

For our numerical experiments, we consider a male patient and a female patient who are Caucasian, do not smoke and were diagnosed with Type 2 diabetes at age 40 with no history of CHD or stroke. Our data set provides insufficient observations for patients older than 80 . Therefore, we set annual decision epochs for a 40-year non-stationary decision horizon, which starts at age 40 , so that treatment decisions are age-dependent prior to age 79 and remain stationary after age 79 based on our infinite-horizon approximation, i.e., $\tau=1, k=0$ and $N=40$. We discretize the continuous range of LR levels into $L=13$ ranges the boundaries of which are presented in Table 1.

Using our clinical dataset we estimate $\widehat{\omega}=0.19815$. We assume $\sigma=0.02$ as our base case (Tsevat et al., 2001, Pignone et al., 2006). In all numerical experiments we assign $\lambda=0.97$ as the annual discount factor, as is common in the health policy and economics literature (Gold et al., 1996). Because the patients' optimal value functions and actions remain stationary beyond age 79 , we use $80+$ in figures and tables to refer to all ages beyond 79 .

We solve our MDP model to find the optimal treatment policies. In Table 2, we present the patients' maximum 
Table 1. LR-range boundaries. Note that ranges are exclusive of their upper bounds.

\begin{tabular}{|c|c|c|c|c|c|c|c|c|c|c|c|c|c|}
\hline \multirow[b]{2}{*}{ Boundary } & \multicolumn{13}{|c|}{ LR-Range } \\
\hline & 1 & 2 & 3 & 4 & 5 & 6 & 7 & 8 & 9 & 10 & 11 & 12 & 13 \\
\hline Lower Bound & 0 & 3 & 3.5 & 4 & 4.5 & 5 & 5.5 & 6 & 6.5 & 7 & 7.5 & 8 & 8.5 \\
\hline Upper Bound & 3 & 3.5 & 4 & 4.5 & 5 & 5.5 & 6 & 6.5 & 7 & 7.5 & 8 & 8.5 & $\infty$ \\
\hline
\end{tabular}

expected QALYs to their first terminal events from ages 40, $50,60,70$ and 80 . Table 2 confirms that the optimal value functions are monotonically nonincreasing in both LR and age. In Table 2 the differences between the optimal value functions range from 2.09 to 2.69 QALYs, which is consistent with gender-based differences in CHD and stroke risks (Pilote et al., 2007). From Table 2, it is also clear that the difference between the patients' optimal value functions in two different LR-ranges increases as they age. The optimal value function of the male patient has a range of 0.77 QALYs at age 40 and 2.58 QALYs at age 80 and beyond. Similarly, the optimal value function of the female patient has a range of 0.69 QALYs at age 40 and increases up to 2.67 QALYs by age 80. Consistent with clinical trials and published risk models, these imply that increased LR levels are more risky, and more significantly affect expected QALYs as patients age.

Figure 2 depicts the optimal LR thresholds to initiate treatment for each patient. For instance, the optimal policy at age 40 can be interpreted as follows: Treatment should be initiated for the male patient if his LR level falls into any range that is higher than 6 . On the other hand, treatment is not recommended at any LR level for the female patient at age 40. Consistent with Proposition 4, the optimal LR thresholds are nonincreasing in age for both patients, which implies that the patients are more likely initiate treatment as they get older. Also, note that the male patient's optimal LR thresholds are never higher than those of the female patient, and the deviation between their optimal LR thresholds are more significant at younger ages. These results are consistent with a recent epidemiological study that has shown that relative cardiovascular risk reduction by treatment is lower for female patients than it is for male patients (Karp et al., 2007). Moreover, they also confirm a meta-analysis of clinical trials for lipid-lowering treatment agents which has demonstrated lesser benefit of treatment for female patients than for male patients (Walsh and Pignone 2004).

\subsection{Sensitivity analysis}

To evaluate the effects of quality-adjustment factor, $\sigma$, and treatment-effect factor, $\omega$, on expected QALYs to first terminal event and optimal policies, we perform one- and two-way sensitivity analyses. Figures 3 and 4 present the optimal treatment policies under various quality-adjustment factors, and illustrate the fact that the optimal threshold for treatment decreases as the disutility of statins decreases.

It is clear that the optimal time to initiate statin treatment is quite sensitive to disutility of using statins. However, for the same disutility level the thresholds of the male patient are never higher than those of the female patient.

In Figure 5, we illustrate the sensitivity of the optimal policies with respect to treatment-effect factor within a $\pm 3 \%$ region around the point estimate for $\omega$. We observe that the thresholds decrease as treatment reduces the patients' LR levels further. It is notable that the male patient has lower treatment thresholds than the female patient even when his response to treatment is lower than that of the female.

In Table 3, we present the results from our two-way sensitivity analyses. An increase in the efficacy of treatment

Table 2. Patients' maximum expected QALYs prior to their first terminal events.

\begin{tabular}{|c|c|c|c|c|c|c|c|c|c|c|c|c|c|c|}
\hline \multirow[b]{2}{*}{ Age } & \multirow[b]{2}{*}{ Gender } & \multicolumn{13}{|c|}{ LR-Range } \\
\hline & & 1 & 2 & 3 & 4 & 5 & 6 & 7 & 8 & 9 & 10 & 11 & 12 & 13 \\
\hline \multirow[t]{2}{*}{$80+$} & Male & 5.93 & 5.69 & 5.47 & 5.29 & 5.08 & 4.92 & 4.74 & 4.59 & 4.47 & 4.35 & 4.26 & 4.01 & 3.65 \\
\hline & Female & 8.41 & 8.13 & 7.92 & 7.66 & 7.45 & 7.22 & 7.05 & 6.89 & 6.73 & 6.52 & 6.40 & 6.18 & 5.74 \\
\hline \multirow[t]{2}{*}{70} & Male & 7.60 & 7.41 & 7.22 & 7.07 & 6.89 & 6.76 & 6.60 & 6.47 & 6.37 & 6.28 & 6.21 & 5.99 & 5.73 \\
\hline & Female & 10.07 & 9.85 & 9.69 & 9.48 & 9.31 & 9.12 & 8.99 & 8.87 & 8.74 & 8.58 & 8.49 & 8.31 & 7.98 \\
\hline \multirow[t]{2}{*}{60} & Male & 11.10 & 10.94 & 10.78 & 10.65 & 10.50 & 10.38 & 10.24 & 10.14 & 10.05 & 9.97 & 9.92 & 9.73 & 9.52 \\
\hline & Female & 13.65 & 13.49 & 13.37 & 13.22 & 13.10 & 12.96 & 12.86 & 12.78 & 12.69 & 12.57 & 12.51 & 12.37 & 12.14 \\
\hline \multirow[t]{2}{*}{50} & Male & 14.87 & 14.75 & 14.63 & 14.54 & 14.42 & 14.34 & 14.23 & 14.16 & 14.10 & 14.04 & 14.01 & 13.87 & 13.73 \\
\hline & Female & 17.43 & 17.32 & 17.23 & 17.12 & 17.04 & 16.94 & 16.88 & 16.82 & 16.76 & 16.69 & 16.65 & 16.56 & 16.41 \\
\hline \multirow[t]{2}{*}{40} & Male & 18.44 & 18.36 & 18.26 & 18.19 & 18.11 & 18.05 & 17.98 & 17.93 & 17.89 & 17.86 & 17.84 & 17.75 & 17.67 \\
\hline & Female & 20.82 & 20.75 & 20.69 & 20.62 & 20.56 & 20.50 & 20.45 & 20.42 & 20.38 & 20.32 & 20.29 & 20.23 & 20.13 \\
\hline
\end{tabular}




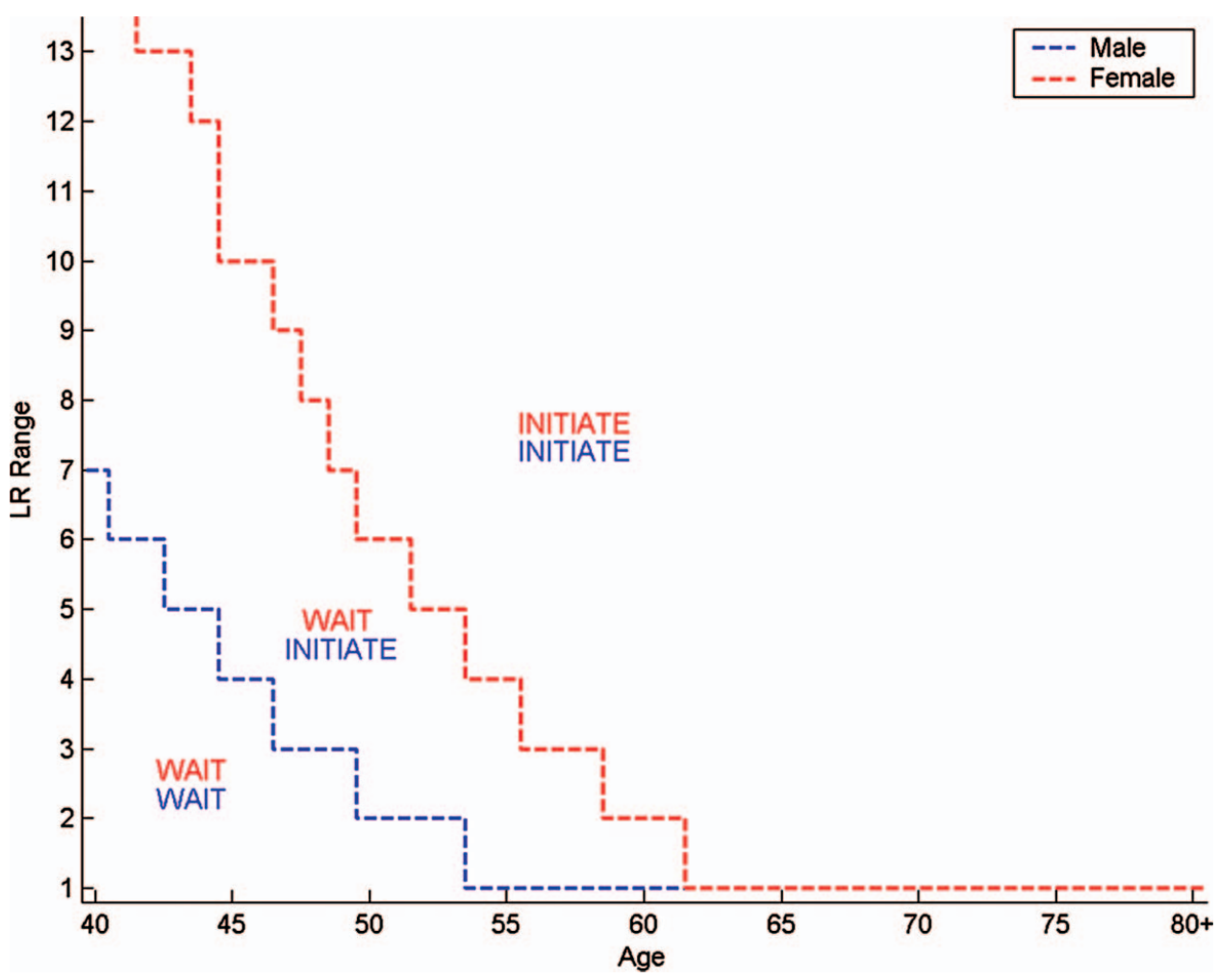

Fig. 2. Optimal LR-range thresholds $\left(\ell_{t}^{*}\right)$ to initiate statin treatment for the base case.

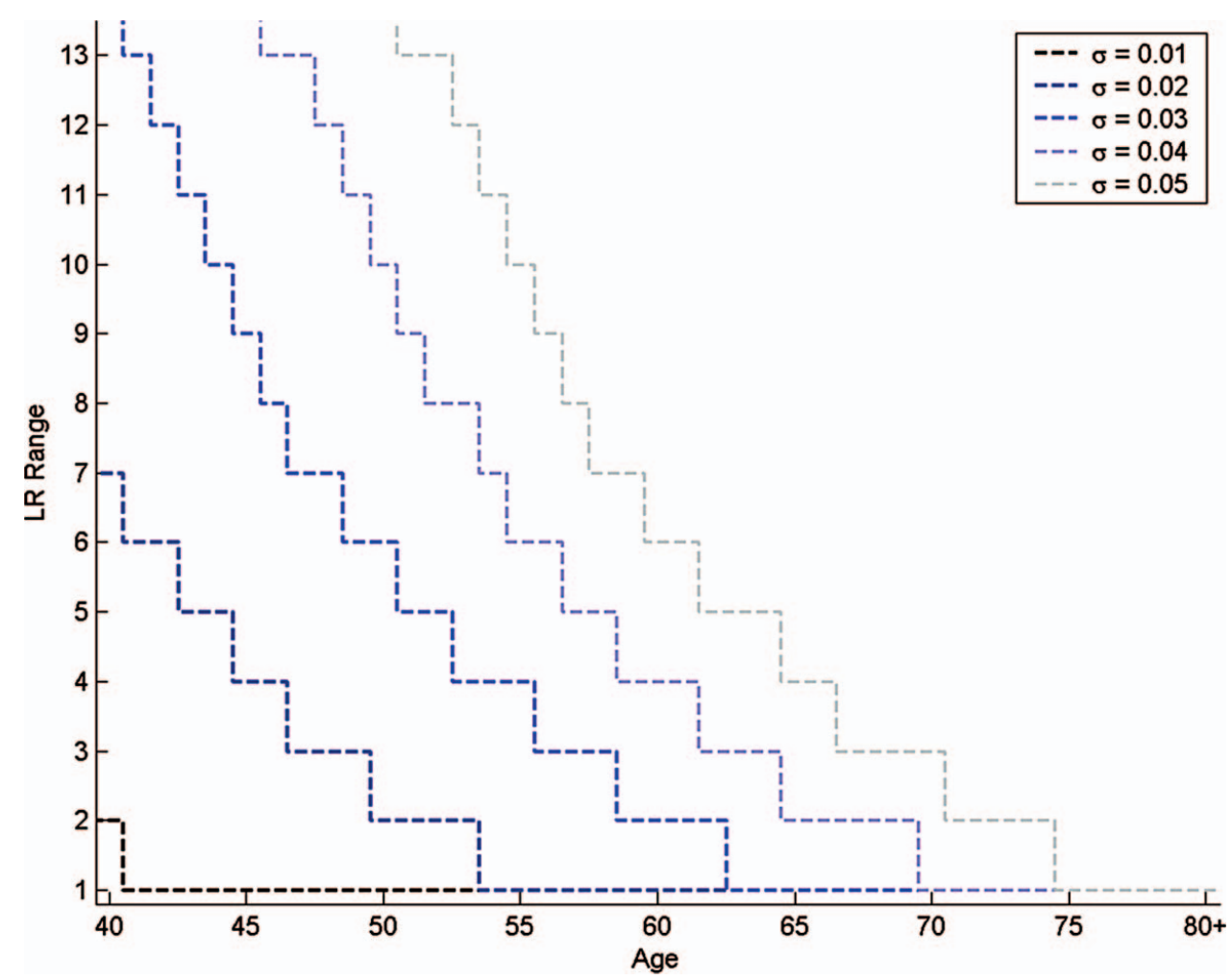

Fig. 3. Sensitivity of the male patient's optimal policy with respect to $\sigma$ for the base case. 


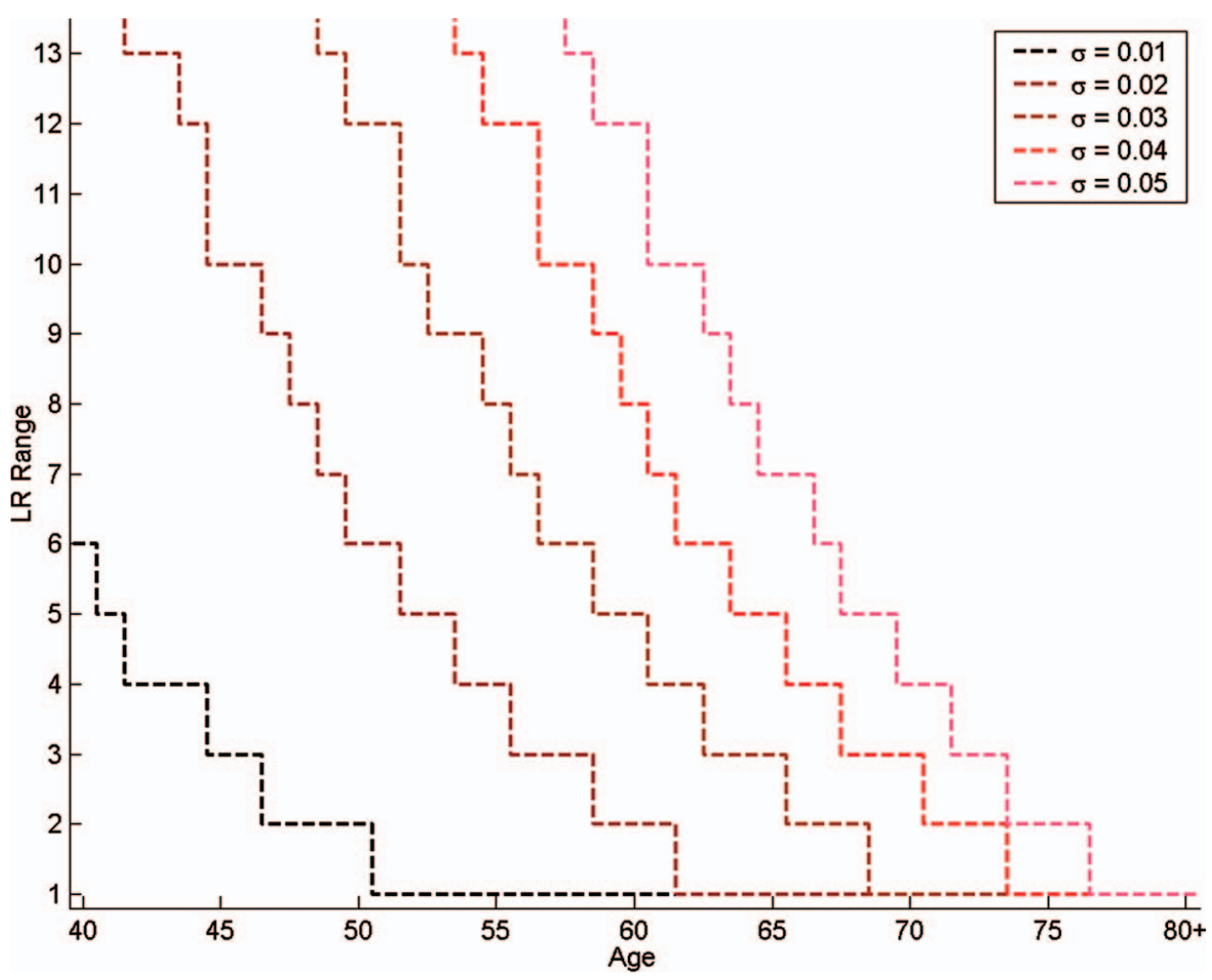

Fig. 4. Sensitivity of the female patient's optimal policy with respect to $\sigma$ for the base case.

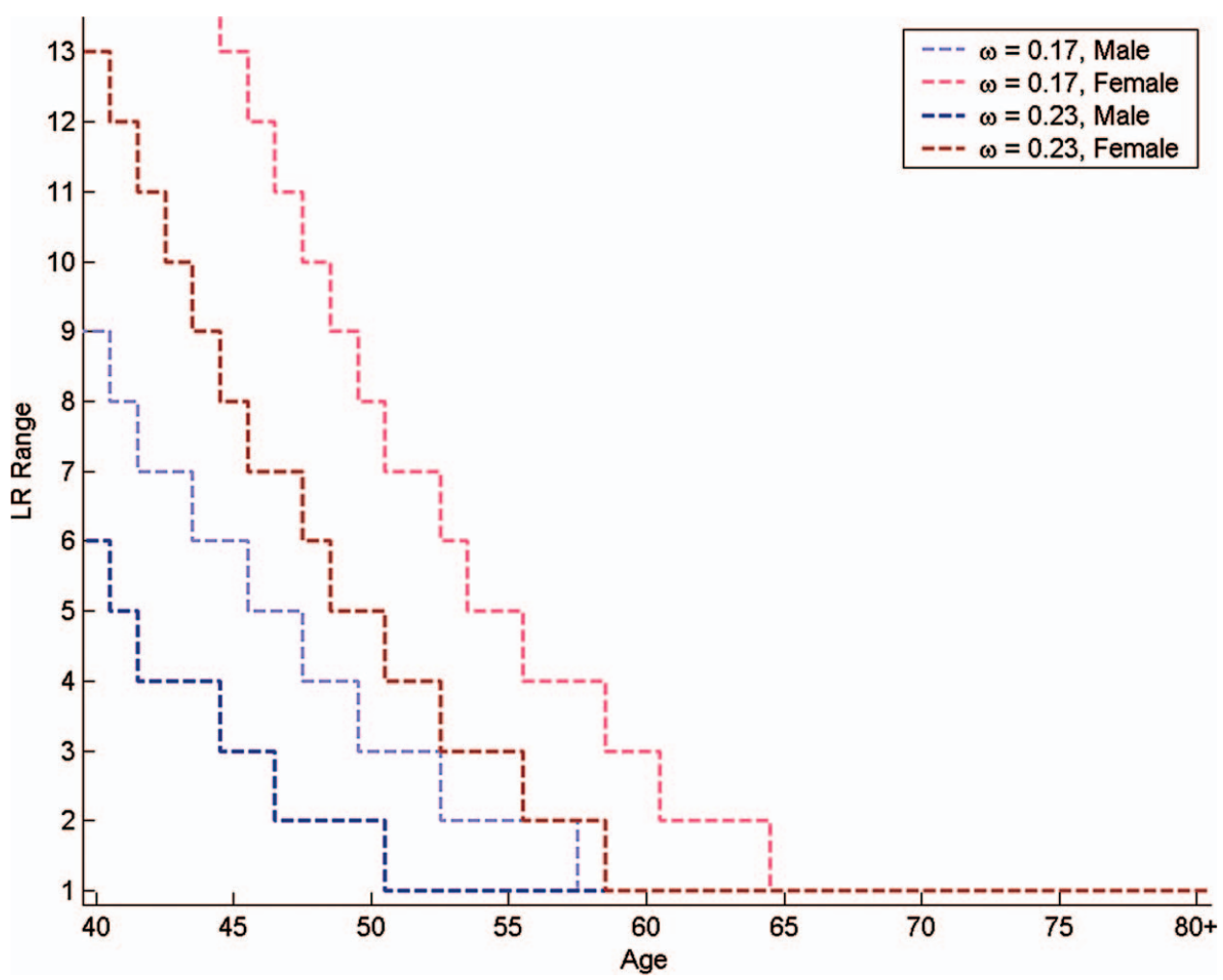

Fig. 5. Sensitivity of the patients' optimal policies with respect to $\omega$ for the base case. 
Table 3. Patients' QALY gains at the time of diagnosis when $\omega$ increases from 0.17 to 0.23 .

\begin{tabular}{|c|c|c|c|c|c|c|c|c|c|c|c|c|c|c|}
\hline \multirow[b]{2}{*}{$\sigma$} & \multirow[b]{2}{*}{ Gender } & \multicolumn{13}{|c|}{ LR-Range } \\
\hline & & 1 & 2 & 3 & 4 & 5 & 6 & 7 & 8 & 9 & 10 & 11 & 12 & 13 \\
\hline \multirow[t]{2}{*}{0.01} & Male & 0.18 & 0.19 & 0.20 & 0.20 & 0.21 & 0.21 & 0.21 & 0.22 & 0.22 & 0.22 & 0.22 & 0.23 & 0.23 \\
\hline & Female & 0.15 & 0.16 & 0.16 & 0.17 & 0.18 & 0.18 & 0.19 & 0.19 & 0.19 & 0.20 & 0.20 & 0.20 & 0.21 \\
\hline \multirow[t]{2}{*}{0.02} & Male & 0.15 & 0.16 & 0.17 & 0.17 & 0.18 & 0.19 & 0.20 & 0.21 & 0.22 & 0.22 & 0.22 & 0.23 & 0.23 \\
\hline & Female & 0.13 & 0.13 & 0.13 & 0.13 & 0.14 & 0.14 & 0.14 & 0.15 & 0.15 & 0.15 & 0.16 & 0.16 & 0.18 \\
\hline \multirow[t]{2}{*}{0.03} & Male & 0.12 & 0.13 & 0.13 & 0.13 & 0.14 & 0.14 & 0.15 & 0.15 & 0.16 & 0.16 & 0.16 & 0.18 & 0.19 \\
\hline & Female & 0.10 & 0.10 & 0.10 & 0.10 & 0.11 & 0.11 & 0.11 & 0.11 & 0.11 & 0.11 & 0.11 & 0.11 & 0.12 \\
\hline
\end{tabular}

Table 4. Patients' QALY gain at the time of diagnosis when $\sigma$ decreases from 0.05 to 0.01 .

LR-Range

\begin{tabular}{llccccccccccccccc}
$\omega$ & Gender & 1 & 2 & 3 & 4 & 5 & 6 & 7 & 8 & 9 & 10 & 11 & 12 & 13 \\
\hline 0.17 & Male & 0.28 & 0.24 & 0.31 & 0.33 & 0.34 & 0.35 & 0.36 & 0.37 & 0.38 & 0.39 & 0.39 & 0.41 & 0.41 \\
& Female & 0.23 & 0.23 & 0.24 & 0.24 & 0.25 & 0.26 & 0.27 & 0.27 & 0.28 & 0.29 & 0.29 & 0.30 & 0.32 \\
0.19815 & Male & 0.34 & 0.36 & 0.38 & 0.39 & 0.41 & 0.42 & 0.44 & 0.45 & 0.46 & 0.46 & 0.47 & 0.48 & 0.49 \\
& Female & 0.27 & 0.28 & 0.29 & 0.30 & 0.31 & 0.32 & 0.33 & 0.34 & 0.34 & 0.35 & 0.36 & 0.37 & 0.39 \\
0.23 & Male & 0.40 & 0.42 & 0.44 & 0.46 & 0.48 & 0.49 & 0.51 & 0.52 & 0.53 & 0.54 & 0.54 & 0.56 & 0.57 \\
& Female & 0.32 & 0.33 & 0.34 & 0.35 & 0.37 & 0.38 & 0.39 & 0.40 & 0.41 & 0.42 & 0.43 & 0.44 & 0.46 \\
\hline
\end{tabular}

Table 5. Patients' expected QALY gains from delaying the initiation of treatment rather than initiating it at the time of diagnosis * $^{*}$ denotes a value $<0.01)$.

\begin{tabular}{|c|c|c|c|c|c|c|c|c|c|c|c|c|c|c|}
\hline \multirow[b]{2}{*}{$\sigma$} & \multirow[b]{2}{*}{ Gender } & \multicolumn{13}{|c|}{ LR-Range } \\
\hline & & 1 & 2 & 3 & 4 & 5 & 6 & 7 & 8 & 9 & 10 & 11 & 12 & 13 \\
\hline \multirow[t]{2}{*}{0.01} & Male & $*$ & $*$ & $*$ & $*$ & $*$ & $*$ & $*$ & * & $*$ & $*$ & * & * & * \\
\hline & Female & 0.02 & 0.01 & 0.01 & $*$ & $*$ & * & $*$ & * & $*$ & $*$ & $*$ & $*$ & * \\
\hline \multirow[t]{2}{*}{0.02} & Male & 0.04 & 0.03 & 0.02 & 0.02 & 0.01 & $*$ & $*$ & $*$ & $*$ & $*$ & $*$ & $*$ & * \\
\hline & Female & 0.11 & 0.10 & 0.09 & 0.08 & 0.07 & 0.06 & 0.05 & 0.05 & 0.04 & 0.03 & 0.03 & 0.02 & * \\
\hline \multirow[t]{2}{*}{0.03} & Male & 0.14 & 0.12 & 0.10 & 0.09 & 0.08 & 0.06 & 0.05 & 0.04 & 0.03 & 0.03 & 0.02 & 0.01 & $*$ \\
\hline & Female & 0.25 & 0.23 & 0.23 & 0.21 & 0.20 & 0.18 & 0.17 & 0.17 & 0.16 & 0.15 & 0.14 & 0.13 & 0.10 \\
\hline \multirow[t]{2}{*}{0.04} & Male & 0.41 & 0.40 & 0.39 & 0.37 & 0.36 & 0.34 & 0.33 & 0.32 & 0.31 & 0.30 & 0.29 & 0.28 & 0.25 \\
\hline & Female & 0.26 & 0.24 & 0.22 & 0.21 & 0.19 & 0.18 & 0.16 & 0.15 & 0.14 & 0.13 & 0.12 & 0.10 & 0.08 \\
\hline \multirow[t]{2}{*}{0.05} & Male & 0.41 & 0.39 & 0.37 & 0.35 & 0.33 & 0.32 & 0.30 & 0.29 & 0.27 & 0.26 & 0.26 & 0.23 & 0.21 \\
\hline & Female & 0.59 & 0.58 & 0.56 & 0.55 & 0.53 & 0.52 & 0.51 & 0.50 & 0.49 & 0.47 & 0.47 & 0.45 & 0.42 \\
\hline
\end{tabular}

Table 6. Patients' expected QALY gains at the time of diagnosis from following the optimal policy rather than ATP III guideline (* denotes a value $<0.01)$.

\begin{tabular}{|c|c|c|c|c|c|c|c|c|c|c|c|c|c|c|}
\hline \multirow[b]{2}{*}{$\sigma$} & \multirow[b]{2}{*}{ Gender } & \multicolumn{13}{|c|}{ LR-Range } \\
\hline & & 1 & 2 & 3 & 4 & 5 & 6 & 7 & 8 & 9 & 10 & 11 & 12 & 13 \\
\hline \multirow[t]{2}{*}{0.01} & Male & 0.03 & 0.01 & 0.01 & * & $*$ & * & $*$ & $*$ & $*$ & $*$ & $*$ & $*$ & * \\
\hline & Female & 0.02 & 0.01 & $*$ & $*$ & $*$ & $*$ & $*$ & $*$ & $*$ & $*$ & $*$ & $*$ & * \\
\hline \multirow[t]{2}{*}{0.02} & Male & 0.07 & 0.09 & 0.09 & 0.08 & 0.07 & 0.06 & 0.05 & 0.05 & 0.04 & 0.03 & 0.03 & 0.02 & 0.01 \\
\hline & Female & 0.01 & 0.02 & 0.02 & 0.01 & 0.01 & * & $*$ & $*$ & $*$ & $*$ & $*$ & $*$ & $*$ \\
\hline \multirow[t]{2}{*}{0.03} & Male & 0.14 & 0.21 & 0.22 & 0.21 & 0.20 & 0.18 & 0.17 & 0.17 & 0.16 & 0.15 & 0.14 & 0.13 & 0.11 \\
\hline & Female & 0.06 & 0.09 & 0.09 & 0.09 & 0.08 & 0.06 & 0.05 & 0.04 & 0.03 & 0.03 & 0.02 & 0.01 & 0.01 \\
\hline \multirow[t]{2}{*}{0.04} & Male & 0.25 & 0.36 & 0.38 & 0.37 & 0.36 & 0.34 & 0.33 & 0.32 & 0.31 & 0.30 & 0.30 & 0.28 & 0.26 \\
\hline & Female & 0.14 & 0.20 & 0.20 & 0.20 & 0.19 & 0.18 & 0.16 & 0.15 & 0.14 & 0.13 & 0.12 & 0.11 & 0.10 \\
\hline \multirow[t]{2}{*}{0.05} & Male & 0.37 & 0.53 & 0.55 & 0.54 & 0.53 & 0.52 & 0.51 & 0.50 & 0.49 & 0.47 & 0.47 & 0.46 & 0.44 \\
\hline & Female & 0.24 & 0.33 & 0.34 & 0.34 & 0.33 & 0.32 & 0.30 & 0.29 & 0.28 & 0.27 & 0.26 & 0.24 & 0.23 \\
\hline
\end{tabular}


Table 7. Patients' expected QALY gains at the time of diagnosis from following the optimal policy rather than modified ATP III guideline $(*$ denotes a value $<0.01)$.

\begin{tabular}{|c|c|c|c|c|c|c|c|c|c|c|c|c|c|c|}
\hline \multirow[b]{2}{*}{$\sigma$} & \multirow[b]{2}{*}{ Gender } & \multicolumn{13}{|c|}{ LR-Range } \\
\hline & & 1 & 2 & 3 & 4 & 5 & 6 & 7 & 8 & 9 & 10 & 11 & 12 & 13 \\
\hline \multirow[t]{2}{*}{0.01} & Male & 0.01 & 0.01 & $*$ & $*$ & * & $*$ & $*$ & $*$ & * & $*$ & $*$ & * & $*$ \\
\hline & Female & 0.02 & 0.01 & 0.01 & $*$ & $*$ & $*$ & $*$ & * & $*$ & $*$ & $*$ & $*$ & * \\
\hline \multirow[t]{2}{*}{0.02} & Male & 0.01 & 0.02 & 0.02 & 0.01 & 0.01 & $*$ & $*$ & $*$ & $*$ & $*$ & $*$ & $*$ & $*$ \\
\hline & Female & 0.05 & 0.09 & 0.09 & 0.08 & 0.07 & 0.06 & 0.05 & 0.05 & 0.04 & 0.03 & 0.03 & 0.02 & 0.01 \\
\hline \multirow[t]{2}{*}{0.03} & Male & 0.06 & 0.10 & 0.09 & 0.09 & 0.08 & 0.06 & 0.05 & 0.04 & 0.03 & 0.03 & 0.02 & 0.01 & 0.01 \\
\hline & Female & 0.14 & 0.22 & 0.22 & 0.21 & 0.20 & 0.18 & 0.17 & 0.17 & 0.16 & 0.15 & 0.14 & 0.13 & 0.11 \\
\hline \multirow[t]{2}{*}{0.04} & Male & 0.13 & 0.20 & 0.20 & 0.20 & 0.19 & 0.18 & 0.16 & 0.15 & 0.14 & 0.13 & 0.12 & 0.11 & 0.10 \\
\hline & Female & 0.26 & 0.37 & 0.38 & 0.37 & 0.36 & 0.34 & 0.33 & 0.32 & 0.32 & 0.30 & 0.30 & 0.28 & 0.26 \\
\hline \multirow[t]{2}{*}{0.05} & Male & 0.23 & 0.33 & 0.34 & 0.34 & 0.33 & 0.32 & 0.30 & 0.29 & 0.28 & 0.27 & 0.26 & 0.24 & 0.23 \\
\hline & Female & 0.40 & 0.55 & 0.55 & 0.54 & 0.53 & 0.52 & 0.51 & 0.50 & 0.49 & 0.47 & 0.47 & 0.46 & 0.44 \\
\hline
\end{tabular}

has greater impact on the optimal value function when the disutility of using statins is lower. When both patients have the same quality-adjustment factor, an increase in the treatment efficacy increases QALYs more for the male patient than the female patient. Regardless of the efficacy of treatment, when the disutility of using statins decreases, the male patient gains more than the female patient. Since increases in expected QALYs with increasing treatment-effect and/or decreasing quality-adjustment factors are greater for higher LR levels, patients that are relatively at higher risk of CHD and stroke benefit more from such changes.

\subsection{Comparison to current treatment guidelines}

In the remainder of this section, we evaluate the performance of three common U.S. guidelines. We consider ATP III guideline, which specifies treatment decisions on the basis of long term CHD risk, and its modification, which sets a tighter thresholds specific to diabetes patients. We also consider recent recommendations from the ADA that call for initiation of statins immediately after the diagnosis. We calculate the patients' expected QALY gains from following our optimal policies rather than these guidelines (see Appendix $\mathrm{C}$ for details on how we estimate expected QALYs under these guidelines). Results for expected QALY gains with respect to ADA, ATP III and modified ATP III guidelines are presented in Tables 5, 6 and 7, respectively. Clearly the gain is lower under ATP III guidelines than under ADA guidelines, owing to the more aggressive use of statins in the latter. From Tables 5, 6 and 7, we observe that the expected QALY gain for the male patient is lower than that for the female patient which we can attribute to the earlier onset of elevated cardiovascular risk in males. We also observe that patients' expected gains increase as the disutility of using statins increases, highlighting the importance of incorporating the effect of disutility of using statins into the treatment decisions.

\section{Conclusions}

Cardiovascular risk models serve as a guide to clinicians for selecting the type of intervention and the aggressiveness of treatment. However, their use in practice has focused on providing raw information about the risk of complications. Unfortunately there has been little direction on how to use this information to make treatment decisions that tradeoff the benefits of risk reduction with the side effects of treatment. We empirically show the importance of tailoring guidelines to short-term CHD and stroke probabilities, and individual patient preferences and responses to statins. We also provide scientific evidence for the threshold-structured treatment policies.

Our study has some limitations. We focus on lipid control, but in practice statin treatment may be combined with other treatments, including blood pressure control medications. We leave the development and the analysis of a more extensive and sophisticated multi-treatment model as a future study. Second, the source data that we use to estimate the LR progression rates belong to a single medical center, and the population that the data belongs to is likely to be healthier than a typical population that may not receive continuous access to health care. Finally, due to limited availability of clinical data, a limitation for our numerical experiments is that we focus only on Caucasian non-smoker patients, and due to possible uncertainties in disutility and treatment effect factors, which we do not include in our model, our results should be cautiously generalized to comment on population-based treatment decisions.

\section{Acknowledgments}

This project was funded in part by the Agency for Healthcare Research and Quality (AHRQ) under R21 Grant 1R21HS017628 (Shah) and by the National Science Foundation under grants CMMI-0844511 and CMMI-0969885 (Denton) and CMMI-0546960 (Kurt, Schaefer). 


\section{References}

Alagoz, O., Hsu, H., Schaefer, A. J., and Roberts, M. S. (2010). Markov decision processes: A tool for sequential decision making under uncertainty. Medical Decision Making, 30(4), 474-483.

Alagoz, O., Maillart, L. M., Schaefer, A. J., and Roberts, M. S. (2004). The optimal timing of living-donor liver transplantation. Management Science, 50(10), 1420-1430.

American Diabetes Association. (2008). Information and data accessed on May 1, 2009, http://www.diabetes.org.

Anderson, K. A., Odel, P. M., Wilson, P. W. F., and Kannel, W. B. (1991). Cardiovascular disease risk profiles. American Heart Journal, 121(2), 293-298.

Barlow, R. E. and Proschan, F. (1965). Mathematical Theory of Reliability. John Wiley \& Sons, New York.

Briel, M., Studer, M., Glass, T. R., and Bucher, H. C. (2004). Effects of statins on stroke prevention in patients with and without coronary heart disease: A meta-analysis of randomized controlled trials. American Journal of Medicine, 117(8) 596-606.

British Cardiac Society, British Hypertension Society, Diabetes UK, HEART UK, Primary Care Cardiovascular Society and The Stroke Association. (2005). JBS 2: Joint British Societies' guidelines on prevention of cardiovascular disease in clinical practice. Heart, 91, $1-52$.

Burt, V. L., Whelton, P., Roccella, E. J., Brown, C., Cutler, J. A., Higgins, M., Horan, M. J., and Labarthe, D. (1995). Prevalence of hypertension in the US adult population : Results from the $3^{r d}$ National Health and Nutrition Examination Survey, 1988-1991. Hypertension, 25(3), 305-313.

Carrera, T., Bonamusa, L., Almirall, L., and Navarro, J. M. (1998). Should age and sex be taken into account in the determination of HbAlc reference range? Diabetes Care, 21(12) 2193-2194.

Colhoun, H., Betteridge, D. J., Durrington, P. N., Hitman, G. A., Neil, H. A., Livingstone, S. J., Thomason, M. J., Mackness, M. I., Charlton-Menys, V., and Fuller, J. H. (2004). Primary prevention of cardiovascular disease with atorvastatin in Type 2 diabetes in the Collaborative Atorvastatin Diabetes Study (CARDS): Multicentre randomised placebo-controlled trial. Lancet, 364(9435) 685-696.

Collins, R., Armitage, J., Parish, S., Sleight, P., and Peto, R. (2002). $\mathrm{MRC} / \mathrm{BHF}$ heart protection study of cholesterol lowering with simvastatin in 20,536 high-risk individuals: A randomised placebocontrolled trial. Lancet, 360(9426), 7-22.

Collins, R., Armitage, J., Parish, S., Sleight, P., and Peto, R. (2003). MRC/BHF heart protection study of cholesterol-lowering with simvastatin in 5963 people with diabetes: A randomised placebocontrolled trial. Lancet, 361(9374), 2005-2016.

Denton, B. T., Kurt, M., Shah, N. D., Bryant, S. C., and Smith, S. A. (2009). Optimizing the start time of statin therapy for patients with diabetes. Medical Decision Making, 29(3), 351-368.

Downs, J. R., Clearfield, M., Weis, S., Whitney, E., Shapiro, D. R., Beere, P. A., and Air Force/Texas Coronary Atherosclerosis Prevention Study Group. (1998). Primary prevention of acute coronary events with lovastatin in men and women with average cholesterol levels: Results of AFCAPS/TexCAPS. Journal of the American Medical Association, 279(20), 1615-1622.

Drummond, M. F., O'Brien, B., Stoddart, G. L., and Torrance, G. W. (1997). Methods for the Economic Evaluation of Health Care Programmes. Oxford University Press, Oxford, England.

Eddy, D. M. and Schlessinger, L. (2003a). Archimedes - a trial-validated model of diabetes. Diabetes Care, 26(11), 3093-3101.

Eddy, D. M. and Schlessinger, L. (2003b). Validation of the Archimedes diabetes model. Diabetes Care, 26(11), 3102-3110.

Fourth Joint Task Force of the European Society of Cardiology. (2007). European guidelines on cardiovascular disease prevention in clinical practice: Executive summary. European Heart Journal, 194(1), $1-45$.
Garber, A. R., Olshen, R. A., Zhang, H., and Venatraman, E.S. (1994). Predicting high risk cholesterol levels. International Statistical Review, 62(2).

Gold, M. R., Patrick, D. L., Torrance, G. W., Fryback, D. G., Hadorn, D. C., Kamlet, M. S., Daniels, N., and Weinstein, M. C. (1996). Identifying and valuing outcomes. Gold, M. R., Siegel, J. E., Russell, L. B., Weinstein, M. C., eds., Cost-Effectiveness in Health and Medicine. Oxford University Press, New York, 82-134.

Gold, M. R., Stevenson, D., and Fryback, D. G. (2002). HALYs and QALYs and DALYs, oh my: Similarities and differences in summary measures of population health. Annu. Rev. Public Health, 23, 115134.

Hajjar, I. and Kotchen, T. A. (2003). Trends in prevalence, awareness, treatment, and control of hypertension in the United States, 19882000. Journal of the American Medical Association, 290(2), 199-206.

Honeycutt, A. A., Boyle, J. P., Broglio, K. R., Thompson, T. J., Hoerger, T. J., Geiss, L. S., and Venkat Narayan, K. M. (2003). A dynamic Markov model for forecasting diabetes prevalence in the United States through 2050. Health Care Management Science, 6(3), 155164.

Kannel, W. B. (1996). Blood pressure as a cardiovascular risk factor. Journal of the American Medical Association, 275(20) 1571-1576.

Karp, I., Chen, S. F., and Pilote, L. (2007). Sex differences in the effectiveness of statins after myocardial infarction. Canadian Medical Association Journal, 176(3), 333-338.

Kilpatrick, E. S., Dominiczak, M. H., and Small, M. (1996). The effects of ageing on glycation and the interpretation of glycaemic control in Type 2 diabetes. QJM: An International Journal of Medicine, 89(4), 307-312.

Kothari, V., Stevens, R. J., Adler, A. I., Stratton, I. M., Manley, S. E., Neil, H. A., and Holman, R. R. (2002). UKPDS 60 - risk of stroke in Type 2 diabetes estimated by the UK Prospective Diabetes Study risk engine. Stroke, 33(7), 1776-1781.

Kreke, J. E., Bailey, M. D., Schaefer, A. J., Roberts, M. S., and Angus, D. C. (2008). Modeling hospital discharge policies for patients with pneumonia-related sepsis. IIE Transactions, 40(9), 861-869.

National Center for Health Statistics (NCHS). (2005). Mortality data from the National Vital Statistics System. Information and data accessed on November 1, 2009, http://www.cdc.gov/ nchs/deaths.htm.

National Cholesterol Education Program. (2001). Executive summary of the 3rd report of the NCEP expert panel on detection, evaluation, and treatment of high blood cholesterol in adults (Adult Treatment Panel III). Journal of the American Medical Association, 285(19), 2486-2497.

National Heart Foundation of Australia, and The Cardiac Society of Australia and New Zealand. (2001). Lipid management guidelines supplement. The Medical Journal of Australia, 175 s57-s88.

New Zealand Guidelines Group. (2003). The assessment and management of cardiovascular risk. Information and data accessed on May 1, 2009, http://www.nzgg.org.nz.

Pasternak, R. C., Smith, S. C., Bairey-Merz, Jr. C. N., Grundy, S. M., Cleeman, J. I., and Lenfant, C. (2002). ACC/AHA/NHLBI clinical advisory on the use and safety of statins. Journal of the American College of Cardiology, 40(3), 567-572.

Phillips, P. S., Haas, R. H., Bannykh, S., Hathaway, N. L., Gray, S., and Kimura, B. J. (2002). Statin-associated myopathy with normal creatine kinase levels. Annals of Internal Medicine, 137(7), 581-585.

Pignone, M., Earnshaw, S., Tice, J. A., and Pletcher, M. J. (2006). Aspirin, statins, or both drugs for the primary prevention of coronary heart disease events in men: A cost-utility analysis. Annals of Internal Medicine, 144(5), 326-336.

Pilote, L., Dasgupta, K., Guru, V., Humphries, K. H., McGrath, J., Norris, C., Rabi, D., Tremblay, J., Alamian, A., Barnett, T., Cox, J., Ghali, W. A., Grace, S., Hamet, P., Ho, T., Kirkland, S., Lambert, M., Libersan, D., O’Loughlin, J., Paradis, G., Petrovich, M., and Tagalakis, V. (2007). A comprehensive view of sex-specific issues 
related to cardiovascular disease. Canadian Medical Association Journal, 176(6) S1-44.

Snow, V., Aronson, M. D., Hornbake, E. R., Mottur-Pilson, C., and Weiss, K. B. (2004). Lipid control in the management of Type 2 diabetes mellitus: A clinical practice guideline from the American College of Physicians. Annals of Internal Medicine, 140(8), 644-649.

Stevens, R. J., Kothari, V., Adler, A. I., Stratton, I. M., and Holman, R. R. (2001). The UKPDS risk engine: A model for the risk of coronary heart disease in Type 2 diabetes (UKPDS 56). Clinical Science, 101(6), 671-679.

Timbie, J. W., R. A. Hayward, and Vijan, S. (2010). Variation in the net benefit of aggressive cardiovascular risk factor control across the US population of patients with diabetes mellitus. Archives of Internal Medicine, 170(12), 1037-1044.

Tsevat, J., Kuntz, K. M., Orav, E. J., Weinstein, M. C., Sacks, F. M., and Goldman, L. (2001). Cost-effectiveness of pravastatin therapy for survivors of myocardial infarction with average cholesterol levels. American Heart Journal, 141(5), 727-734.

Turner, R. C. (1998). The UK Prospective Diabetes Study - a review. Diabetes Care, 21 C35-C38.

UKPDS Group. (1998). Intensive blood glucose control with sulphonylureas or insulin compared with conventional treatment and risk of complications in patients with Type 2 diabetes (UKPDS 33). Lancet, 352(6), 837-853.

Valdez-Flores, C. and Feldman, R. M. (1989). A survey of preventive maintenance models for stochasically deteriorating single-unit systems. Naval Research Logistics, 36(4) 419-446.

Vijan, S. and Hayward, R. A. (2004). Pharmacologic lipid-lowering therapy in Type 2 diabetes mellitus: Background paper for the American College of Physicians. Annals of Internal Medicine, 140(8), 650-658.

Walsh, J. M. E. and Pignone, M. (2004). Drug treatment of hyperlipidemia in women. Journal of the American Medical Association, 291(18), 2243-2252.

Wannamethee, S. G., Shaper, A. G., and Ebrahim, S. (2000). HDLcholesterol, total cholesterol, and the risk of stroke in middle-aged British men. Stroke, 31(8) 1882-1888.

\section{Appendix A: Estimating CHD and Stroke Probabilities}

We briefly summarize how the UKPDS risk model predicts the CHD and stroke probabilities of a patient who was diagnosed with Type 2 diabetes $k$ years before epoch 0 with no abnormal heart rhythm due to fibrillation of atrial heart muscles. We use the CHD probability equation of Stevens et al. (2001) and stroke probability equation of Kothari et al. (2004). For $(\ell, m) \in \mathcal{L}^{\prime} \times \mathcal{M}$ and $t \in T^{\prime}$, we define the probabilities $\pi_{t}^{C}(\ell, m)$ and $\pi_{t}^{S}(\ell, m)$ by the following equations:

$$
\begin{aligned}
& \pi_{t}^{C}(\ell, m)=1-\exp \left\{-\eta_{t}^{C}(\ell, m)\left(\delta_{C}\right)^{k+t \tau}\left(\frac{1-\left(\delta_{C}\right)^{\tau}}{1-\delta_{C}}\right)\right\}, \\
& \pi_{t}^{S}(\ell, m)=1-\exp \left\{-\eta_{t}^{S}(\ell, m)\left(\delta_{S}\right)^{k+t \tau}\left(\frac{1-\left(\delta_{S}\right)^{\tau}}{1-\delta_{S}}\right)\right\} ;
\end{aligned}
$$

where, $\delta_{C} \geq 0$ and $\delta_{S} \geq 0$ are constant and identical for all patients, and

$$
\begin{aligned}
\eta_{t}^{C}(\ell, m)= & \beta_{0} \beta_{1}^{(\text {Age-55) }} \beta_{2}^{\text {Gender }} \beta_{3}^{\text {Ethnicity }} \beta_{4}^{\text {Smoking }} \beta_{5}^{\text {Hb Alc }(t)-6.72} \\
& \times \beta_{6}^{[S B P(t)-135.7] / 10} \beta_{7}^{\ln \left[(1-m \omega) L R_{\ell}\right]-1.59}, \quad \text { (2a) }
\end{aligned}
$$

$$
\begin{aligned}
\eta_{t}^{S}(\ell, m)= & \gamma_{0} \gamma_{1}^{(\text {Age }-55)} \gamma_{2}^{\text {Gender }} \gamma_{3}^{\text {Smoking }} \gamma_{4}^{[S B P(t)-135.5] / 10} \\
& \times \gamma_{5}^{(1-m \omega) L R_{\ell}-5.11}
\end{aligned}
$$

In (2a) and (2b), $\beta_{0}$ and $\gamma_{0}$ are referred as intercepts, and each $\beta_{i}, i=1, \ldots, 7$, and $\gamma_{j}, j=1, \ldots, 5$, is a nonnegative parameter and called the risk ratio for the risk factor appearing as its exponent. Of these risk factors, $L R_{\ell}$ denotes the patient's off-treatment LR in state $\ell \in \mathcal{L}^{\prime}$, and $H b A 1 c(t)$ $(\%)$ and $S B P(t)(\mathrm{mmHg})$ denote the patient's HbAlc and SBP levels at epoch $t$, respectively, and Age, Gender, Ethnicity and Smoking are static variables with values determined as follows:

- Age: Age when diagnosed.

- Gender: 1 for female; 0 for male.

- Ethnicity: 1 for Afro-Caribbean; 0 for Caucasian or Asian-Indian.

- Smoking: 1 for a smoker at diagnosis of the disease; 0 , otherwise.

Consistent with the results of randomized clinical trials and the parameter estimates of Stevens et al. (2001) and Kothari et al. (2004), we assume that $\beta_{7}, \gamma_{5} \geq 1$, i.e., LR is a positive risk factor for a CHD or a stroke event. More explicitly, given all factors other than the patient's cholesterol levels are fixed, an increase in the patient's LR levels does not decrease the patient's CHD and stroke probabilities.

\section{Appendix B: Data Sources and Parameter Estimation}

We estimate the transition probabilities of our model using the Mayo Clinic Diabetes Electronic Management System (DEMS) data Gorman et al. (2000), and NCHS mortality rate tables (2009). DEMS consists of longitudinal medical records for patients with Type 2 diabetes at Mayo Clinic. The patient data in DEMS are available quarterly with detailed treatment information and laboratory measurements for TC, HDL, SBP, HbA1c and triglyceride levels. The majority of the patients in DEMS are non-smoker Caucasian patients with no prior CHD or stroke and no abnormal heart rhythm due to fibrillation of atrial heart muscles at the age of diagnosis. Therefore, we limit our study to such patients with data in the years 1993-2005, creating a cohort of $M=663$ patients. To maximize the utilization of sparsely available data, we estimate incomplete data points and obtain complete sequences of quarterly available TC and HDL levels. We fit cubic splines to each patient's sequence of TC and HDL measurements in an approach similar to that of Shechter (2006).

The effects of using statins on patient's LR levels have been reported as relative reductions in patients' TC and HDL levels in clinical trials (Herbert et al. 1997; Maron et al. 2000). Therefore, we estimate the patients' treatment effect factors implicitly through relative reductions in their TC and HDL levels, and assume that using statins 
changes the patients' current TC and HDL levels by factors $c$ and $h$, respectively, where $0<c<1$ and $h>1$. To estimate $c$ and $h$, since the decision of initiating treatment is revisited annually, we observe the treatment's impact on TC and HDL levels by focusing on 6-month intervals immediately before and after the initiation of treatment. Although it is not clinically recommended (Snow et al. 2004; Vijan and Hayward 2004), in the DEMS data set we observe some patients who gave up using statins and have initiated treatment more than once. We consider all initiations and re-initiations to estimate $c$ and $h$. We let $K(i)$ denote the number of times that patient $i$ initiated treatment for $i=1, \ldots, M$. Then we let the pairs $\left[v_{T C}^{i}(j, k, 0), v_{H D L}^{i}(j, k, 0)\right]$ and $\left[v_{T C}^{i}(j, k, 1), v_{H D L}^{i}(j, k, 1)\right]$ denote the $i$ th patient's total cholesterol and HDL levels $j$ quarters before and after the $k$ th initiation of treatment, respectively, for $i=1, \ldots, M, j=1,2$, and $k=1, \ldots, K(i)$, and estimate $c$ and $h$ by the following formulae:

$$
\widehat{c}=\frac{\sum_{i=1}^{M} \frac{1}{K(i)} \sum_{k=1}^{K(i)} \frac{\sum_{j=1}^{2} v_{T C}^{i}(j, k, 1)}{\sum_{j=1}^{2} v_{T C}^{i}(j, k, 0)}}{M}
$$

and

$$
\widehat{h}=\frac{\sum_{i=1}^{M} \frac{1}{K(i)} \sum_{k=1}^{K(i)} \frac{\sum_{j=1}^{2} v_{H D L}^{i}(j, k, 1)}{\sum_{j=1}^{2} v_{H D L}^{i}(j, k, 0)}}{M} .
$$

In (3), each ratio term within the inner sum of the numerator corresponds to the ratio of the average TC or HDL level within 6 months after an initiation of treatment to that within 6 months before that initiation. Using (3) over the spline-fitted data we estimate $\widehat{c}=0.86026$ and $\widehat{h}=1.07284$, respectively, which imply a $19.815 \%$ reduction in LR levels by treatment, i.e., $\widehat{\omega}=1-\widehat{c} / \widehat{h}=0.19815$.

To estimate the conditional transition probabilities among the LR-ranges for each gender, we use the patients' off-treatment LR levels. We calculate a corresponding offtreatment LR level for each spline-fitted on-treatment LR level. We assume $1 / \widehat{c}$ and $1 / \widehat{h}$ denote the factors for the inverse effects of statin treatment on TC and HDL levels; that is, stopping using statins changes the level of TC by a factor of $1 / \widehat{c}$, and the level HDL by a factor of $1 / \widehat{h}$. By using the estimates $1 / \widehat{c}$, and $1 / \widehat{h}$, we normalize the patients' spline-fitted on-treatment TC and HDL levels so that we obtain sequences of TC and HDL estimates in which all patients are assumed to be off-treatment. We calculate the off-treatment LR levels over these sequences and discretize the continuous range of LR levels into $L=13$ ranges. We let state $\ell \in \mathcal{L}^{\prime}$ refer to range $\left[L B_{L R}(\ell), U B_{L R}(\ell)\right)$, where $L B_{L R}(\ell)$ and $U B_{L R}(\ell)$ denote the respective lower and upper bounds of the range $\ell$. For each patient, we calculate the total number of off-treatment LR estimates in each range in all quarters and ages. Then, we count the number of transitions from each range to all other ranges between the same quarters of successive ages, and compute the gender-specific conditional LR transition probabilities. We formalize this process for male patients as follows: We let $b_{T C}^{i}(t, j)$ and $b_{H D L}^{i}(t, j)$ denote the off-treatment TC and HDL values of male patient $i$ in the $j^{\text {th }}$ quarter of age $t$ for $i=1, \ldots, M_{m}, j=1, \ldots, 4$ and $t=40, \ldots, 80$, respectively, where $M_{m}$ is the number of male patients in the data set. We define two indicator functions: $\psi(z)=\left\{\ell \in \mathcal{L}^{\prime}: z \in\left[L B_{L R}(\ell), U B_{L R}(\ell)\right)\right\}$, and

$$
I_{t, j}^{i}(\ell)= \begin{cases}1 & \text { if } \psi\left(b_{T C}^{i}(t, j) / b_{H D L}^{i}(t, j)\right)=\ell, \\ 0 & \text { otherwise }\end{cases}
$$

for $i=1, \ldots, M_{m}, j=1, \ldots, 4, t=40, \ldots, 80$ and $\ell, \ell^{\prime} \in \mathcal{L}^{\prime}$. Then the transition probability between ranges $\ell, \ell^{\prime} \in \mathcal{L}^{\prime}$ is estimated as:

$$
\begin{aligned}
q\left(\ell^{\prime} \mid \ell\right)= & \sum_{i=1}^{M_{m}} \sum_{j=1}^{4} \sum_{t=40}^{79} I_{t, j}^{i}(\ell) I_{t+1, j}^{i}\left(\ell^{\prime}\right) / \\
& \left(\sum_{i=1}^{M_{m}} \sum_{j=1}^{4} \sum_{t=40}^{79} \sum_{\ell^{\prime} \in \mathcal{L}^{\prime}} I_{t, j}^{i}(\ell) I_{t+1, j}^{i}\left(\ell^{\prime}\right)\right) .
\end{aligned}
$$

We calculate gender-specific LR averages in each range and assign them as the corresponding values of $\mathrm{LR}_{\ell}$. Finally, because our model assumes deterministic evolution of the patient's SBP and HbA1c levels, we use gender-specific SBP and HbA1c estimates from Denton et al. (2009), and use the NCHS (2001) mortality rate tables to estimate the patients' non-CHD or non-stroke-related death probabilities.

\section{Appendix C: Calculating Expected QALYs under ATP III Guidelines}

ATP III guideline and its variants (NCEP 2001) categorize the patients with respect to their 10-year CHD risks and set a specific LDL target for each of these categories. If a patient's LDL level exceeds the LDL threshold that is set for her CHD risk category, then she is considered for statin therapy. For instance, if the patient's 10-year CHD risk is below $10 \%$ and her LDL is above $100 \mathrm{mg} / \mathrm{dL}$, then she should initiate statins (Table 1 summarizes ATP III guidelines).

Therefore, to estimate the patients' expected QALYs prior to their first terminal events under ATP III guidelines we need to assess their off-treatment 10-year CHD probabilities and LDL levels. We let $\varphi_{t}(\ell)$ denote the patient's probability of incurring a CHD event within the next 10 years when she is in state $\ell \in \mathcal{L}^{\prime}$ at epoch $t \in T^{\prime}$ and by the CHD probability equation of Stevens et al. (2001) we calculate it as:

$$
\varphi_{t}(\ell)=1-\exp \left\{-\eta_{t}^{C}(\ell, 0)\left(\delta_{C}\right)^{k+t \tau}\left(\frac{1-\left(\delta_{C}\right)^{10}}{1-\delta_{C}}\right)\right\}
$$


Table 1. Description of ATP III guidelines (LDL units are in $\mathrm{mg} / \mathrm{dL})$.

\begin{tabular}{ll}
\hline Guideline & \multicolumn{1}{c}{ Treatment Policy } \\
\hline ATP III & Treat based on long-term risk-level-based \\
& LDL targets \\
& 10-year CHD Risk $\geq 20 \%$ and LDL $>100$, \\
& 10-year CHD Risk in between 10-20\% and \\
& LDL $>160$, \\
& 10-year CHD Risk $<10 \%$ and LDL $>190$ \\
Modified ATP & Consider diabetes as a cardiovascular risk \\
III & equivalent for treatment \\
& Consider everyone as high risk and, \\
& Treat according to ATP III guideline if LDL \\
& exceeds 100
\end{tabular}

where $\eta_{t}^{C}(\ell, 0)$ is defined by (2a). In general, physicians do not measure the patients' LDL levels directly, but rather estimate them implicitly by the Friedewald's equation using TC, HDL and triglycerides (Warnick et al. 1990). Because LR is defined only in terms of the patient's TC and HDL levels, we estimate the patients' triglyceride levels only as a function of age. We estimate the patients' yearly triglyceride levels by first fitting cubic splines to their incomplete data and taking the average observation of each age over the spline-fitted data of the whole cohort. We let $T R_{t}$ denote the patient's triglyceride level at epoch $t \in T^{\prime}$.

Because the patient's HDL is uncertain with respect to her LR, we define an LR-range-specific probability mass function for her HDL. We discretize the continuous range of HDL levels into 14 ranges where each $h \in \mathcal{H}=\{1,2, \ldots, 14\}$ refers to an HDL-range. We define $D(h \mid \ell)$ to be the patient's probability of having an HDL in range $h \in \mathcal{H}$ given her LR is in range $\ell \in \mathcal{L}^{\prime}$. Because LR is the ratio of the patient's TC to her HDL, we count the total number of HDL estimates in each HDL-range corresponding to each specific LR-range and estimate the probability $D(h \mid \ell)$ by dividing the number of HDL estimates in HDL-range $h \in \mathcal{H}$ to the total number of estimates in all HDL-ranges corresponding to range $\ell \in \mathcal{L}^{\prime}$. The formalization of this process is similar to that of estimating the conditional LR-transition probabilies. To compute the patients' LDL levels, we calculate $\mathrm{HDL}_{h}$ as the gender-specific HDL average in HDL-range $h \in \mathcal{H}$. Then, we let $L D L_{t}(\ell, h)$ denote the LDL of a patient with an LR in range $\ell \in \mathcal{L}^{\prime}$ and HDL in range $h \in \mathcal{H}$ at epoch $t \in T^{\prime}$, and calculate it by the Friedewald's equation as $L D L_{t}(\ell, h)=H D L_{h}\left(L R_{\ell}-1\right)-0.2 T R_{t}$ for $\ell \in \mathcal{L}^{\prime}, h \in \mathcal{H}$ and $t \in T^{\prime}$. To compute the patients' expected QALYs prior to their first terminal events under ATP III and modified ATP III guidelines, we define $d_{t}(\ell, h)$ as the decision recommended by the guideline in LDL-range $\ell \in \mathcal{L}^{\prime}$ and HDL- range $h \in \mathcal{H}$ at epoch $t \in T^{\prime}$; that is,

$$
d_{t}(\ell, h)=\left\{\begin{array}{c}
I \quad \text { if } \varphi_{t}(\ell) \geq 0.2 \text { and } L D L_{t}(\ell, h) \geq 100 \mathrm{mg} / \mathrm{dL}, \\
\quad \text { or } \varphi_{t}(\ell) \geq 0.1 \text { and } L D L_{t}(\ell, h) \\
\geq 160 \mathrm{mg} / \mathrm{dL}, \text { or } L D L_{t}(\ell, h) \geq 190 \mathrm{mg} / \mathrm{dL}, \\
W \quad \text { otherwise, }
\end{array}\right.
$$

for ATP III guideline and,

$$
d_{t}(\ell, h)= \begin{cases}I & \text { if } L D L_{t}(\ell, h) \geq 100 \mathrm{mg} / \mathrm{dL}, \\ W & \text { otherwise }\end{cases}
$$

for modified ATP III guideline, respectively.

Since the patient's HDL is implicit with respect to her LR, in our modeling framework, the decision recommended by the guidelines is probabilistic with respect to her LR-range. We define $\alpha_{t}(\ell)=\sum_{\left\{h: d_{t}(\ell, h)=I\right\}} D(h \mid \ell)$ as the probability of initiating treatment in range $\ell \in \mathcal{L}^{\prime}$ at epoch $t \in T^{\prime}$ under each of the aforementioned guidelines. Then, for each of the guidelines, we let $\vartheta_{t}(\ell)$ denote the patient's expected QALYs prior to her first terminal event in range $\ell \in \mathcal{L}^{\prime}$ at epoch $t \in T^{\prime}$ and recursively calculate it as:

$$
\vartheta_{t}(\ell)\left\{\begin{array}{r}
\alpha_{t}(\ell) \mu_{t}(\ell)+\left[1-\alpha_{t}(\ell)\right]\left[r^{0}(\ell)+\lambda \sum_{\ell^{\prime} \in \mathcal{L}^{\prime}} p_{t}^{0}\left(\ell^{\prime} \mid \ell\right) \vartheta_{t+1}\left(\ell^{\prime}\right)\right] \\
\text { for } \ell \in \mathcal{L}^{\prime} \text { and } t<N, \\
\alpha_{t-1}(\ell) \mu_{t}(\ell)+\left[1-\alpha_{t-1}(\ell)\right]\left[r^{0}(\ell)+\lambda \sum_{\ell^{\prime} \in \mathcal{L}^{\prime}} p_{t-1}^{0}\left(\ell^{\prime} \mid \ell\right) \vartheta_{t}\left(\ell^{\prime}\right)\right] \\
\text { for } \ell \in \mathcal{L}^{\prime} \text { and } t=N .
\end{array}\right.
$$

\section{Appendix D: Proofs of Propositions}

In this appendix, we present the proofs of mathematical statements. Throughout the appendix, we let the terms in bold refer to a real-valued $L+1$ dimensional vector, i.e., $\mathbf{v}$ refers to the vector $[v(\ell)]_{\ell \in \mathcal{L}}$. For convenience, given $\mathbf{v} \in \mathbb{R}^{L+1}, \ell \in \mathcal{L}, m \in\{0,1\}$ and $t \in T^{\prime}$, we let $F_{t}^{m}(\ell, \mathbf{v})=\lambda \sum_{\ell^{\prime} \in \mathcal{L}} p_{t}^{m}\left(\ell^{\prime} \mid \ell\right) v\left(\ell^{\prime}\right)$. We also represent componentwise operations and relations between two vectors in vector notation. For instance, given $\mathbf{v}_{1}=\left[v_{1}(\ell)\right]_{\ell \in \mathcal{L}}$ and $\mathbf{v}_{2}=\left[v_{2}(\ell)\right]_{\ell \in \mathcal{L}}, \mathbf{v}_{1}=\mathbf{v}_{2}$ refers to $v_{1}(\ell)=v_{2}(\ell)$ for all $\ell \in \mathcal{L}$.

In the proofs that we proceed by induction on the iterates of the value iteration algorithm at epoch $t=N$, for $\ell \in \mathcal{L}$ we let $u_{N}^{k}(\ell)$ and $\mu_{N}^{k}(\ell)$ denote the associated values of $u_{N}(\ell)$ and $\mu_{N}(\ell)$ at iteration $k$, respectively. More explicitly, for $\ell \in \mathcal{L}$ and $k \geq 0$, we define $\mu_{N}^{k+1}(\ell)=r^{1}(\ell)+F_{N-1}^{1}\left(\ell, \mu_{N}^{k}\right)$ and $u_{N}^{k+1}(\ell)=\max \left\{r^{0}(\ell)+F_{N-1}^{0}\left(\ell, \mathbf{u}_{N}^{k}\right), \mu_{N}(\ell)\right\}$. Because the state space and rewards are finite and, $\lambda<1$, if $\boldsymbol{\mu}_{N}^{0}$ and $\mathbf{u}_{N}^{0}$ are finite, by Theorem 6.3.1 of Puterman [8], $\lim _{k \rightarrow \infty} \mu_{N}^{k}(\ell)=\mu_{N}(\ell)$ and $\lim _{k \rightarrow \infty} u_{N}^{k}(\ell)=u_{N}(\ell)$ for all $\ell \in \mathcal{L}$. Because $L+1$ is an absorbing state which does not provide any rewards, $u_{N}^{k}(L+1)=\mu_{N}^{k}(L+1)=0$ at each iteration $k$ of the value iteration algorithm. Therefore, $u_{N}(L+1)=\mu_{N}(L+1)=0$. Also, by backwards induction on $t \in T$, it can be observed that $u_{t}(L+1)=$ $\mu_{t}(L+1)=0$ for all $t \in T$. 
Proof. of Proposition 1: Because $\mathbf{P}^{1}(t)$ is IFR for all $t \in T^{\prime}$ and $r^{1}(\ell)$ is nonincreasing in $\ell \in \mathcal{L}$, by the infinite-horizon extension of Theorem 4.7.3 of Puterman (1994), $\mu_{N}(\ell)$ is nonincreasing in $\ell \in \mathcal{L}$. Then, Theorem 4.7.3 of Puterman [8] itself implies that $\mu_{t}(\ell)$ is nonincreasing in $\ell \in \mathcal{L}$ for all $t \in T^{\prime}$. Because $r^{0}(\ell)$ is nonincreasing in $\ell \in \mathcal{L}$ and $\mu_{t}(\ell)$ is nonincreasing in $\ell \in \mathcal{L}$ for all $t \in T^{\prime}$, the proof of the monotonicity of $u_{t}(\ell)$ is similar by applying Theorem 4.7.3 of Puterman (1994) and its infinite-horizon extension.

Proof. of Proposition 2: It is sufficient to show that $u_{t}(\ell)-$ $\mu_{t}(\ell)$ is nonincreasing in $\ell \in \mathcal{L}$ for all $t \in T$. For $t=N$, we will prove the result by induction on the iterates of the value iteration algorithm. Initialize $\mathbf{u}_{N}^{0}=\boldsymbol{\mu}_{N}$, and for some $n \geq 0$, suppose $u_{N}^{k}(\ell)-\mu_{N}(\ell)$ is nonincreasing in $\ell \in \mathcal{L}$. Then, for any arbitrary $\ell \in \mathcal{L}^{\prime}$, consider the two possible cases for $u_{N}^{n+1}(\ell)-u_{N}^{n+1}(\ell+1)$.

1. If $u_{N}^{n+1}(\ell+1)=\mu_{N}(\ell+1)$, then $u_{N}^{n+1}(\ell)-u_{N}^{n+1}(\ell+$ 1) $\geq \mu_{N}(\ell)-\mu_{N}(\ell+1)$.

2. If $u_{N}^{n+1}(\ell+1)>\mu_{N}(\ell+1)$, then

$$
\begin{aligned}
u_{N}^{n+1}(\ell)-u_{N}^{n+1}(\ell+1) \geq & F_{N-1}^{0}\left(\ell, \mathbf{u}_{N}^{n}\right)-F_{N-1}^{0}\left(\ell+1, \mathbf{u}_{N}^{n}\right) \\
\geq & F_{N-1}^{0}\left(\ell, \mu_{N}\right)-F_{N-1}^{0}\left(\ell+1, \mu_{N}\right) \\
\geq & \mu_{N-1}(\ell)-\mu_{N-1}(\ell+1)=\mu_{N}(\ell) \\
& -\mu_{N}(\ell+1) .
\end{aligned}
$$

Since $\mathbf{P}^{0}(N-1)$ is IFR, by the induction hypothesis, Lemma 4.7.2 of Puterman (1994) implies $F_{N-1}^{0}\left(\ell, \mathbf{u}_{N}^{n}-\right.$ $\left.\boldsymbol{\mu}_{N}\right) \geq F_{N-1}^{0}\left(\ell+1, \mathbf{u}_{N}^{n}-\boldsymbol{\mu}_{N}\right)$ and this yields the first inequality in (5). The second inequality follows from the assumption that $B_{N-1}(\ell)$ is nondecreasing in $\ell \in \mathcal{L}^{\prime}$. Then, since $\boldsymbol{\mu}_{N-1}=\boldsymbol{\mu}_{N}$, the equality in (5) follows.

Thus, $u_{N}^{n+1}(\ell)-\mu_{N}(\ell)$ is nonincreasing in $\ell \in \mathcal{L}$. Then, the convergence of the algorithm implies that $u_{N}(\ell)-$ $\mu_{N}(\ell)$ is nonincreasing in $\ell \in \mathcal{L}$. The rest of the proof is similar by backwards induction on $t \in T$.

Proof. of Proposition 3: First, by backwards induction on $t \in T$, we will show that $\mu_{t}(\ell)$ is nonincreasing in $t \in T$ for all $\ell \in \mathcal{L}$. By definition, we have $\boldsymbol{\mu}_{N-1}=\boldsymbol{\mu}_{N}$. As the induction hypothesis, for some $k+1<N-1$, assume that $\boldsymbol{\mu}_{k+1} \geq \boldsymbol{\mu}_{k+2}$. Then, for an arbitrary $\ell \in \mathcal{L}^{\prime}$ :

$$
\begin{aligned}
\mu_{k}(\ell)-\mu_{k+1}(\ell) & =F_{k}^{1}\left(\ell, \mu_{k+1}\right)-F_{k+1}^{1}\left(\ell, \mu_{k+2}\right) \\
& \geq F_{k+1}^{1}\left(\ell, \mu_{k+1}-\mu_{k+2}\right) \geq 0 .
\end{aligned}
$$

Since $p_{t+1}^{1}(L+1 \mid \ell) \geq p_{t}^{1}(L+1 \mid \ell)$ for all $\ell \in \mathcal{L}$ and $t \in T^{\prime} \backslash$ $\{N-1\}$, by definition, $p_{k}^{1}\left(\ell^{\prime} \mid \ell\right) \geq p_{k+1}^{1}\left(\ell^{\prime} \mid \ell\right)$ for all $\ell^{\prime} \in \mathcal{L}^{\prime}$ and the first inequality in (6) follows. Then, the second inequality in (6) follows from the induction hypothesis. Hence, $\boldsymbol{\mu}_{k} \geq \boldsymbol{\mu}_{k+1}$, and by induction $\mu_{t}(\ell)$ is nonincreasing in $t \in T$ for all $\ell \in \mathcal{L}$. By this fact, the proof of the monotonicity of $u_{t}(\ell)$ in $t \in T$ is similar.

Proof. of Proposition 4: It is sufficient to show that $u_{t}(\ell)-$ $\mu_{t}(\ell)$ is nonincreasing in $t \in T$ for all $\ell \in \mathcal{L}$, which we will prove by backwards induction on $t$. By definition, $\boldsymbol{\mu}_{N-1}=$
$\boldsymbol{\mu}_{N}$. By definition, we also have $\mathbf{u}_{N-1}=\mathbf{u}_{N}$. Therefore, $\mathbf{u}_{N}-$ $\boldsymbol{\mu}_{N}=\mathbf{u}_{N-1}-\boldsymbol{\mu}_{N-1}$. As the induction hypothesis, for some $k+1<N$, suppose $\mathbf{u}_{k+1}-\boldsymbol{\mu}_{k+1} \geq \mathbf{u}_{k+2}-\boldsymbol{\mu}_{k+2}$. Now, for fixed $\ell \in \mathcal{L}^{\prime}$ consider the possible cases for $u_{k}(\ell)-u_{k+1}(\ell)$.

1. If $u_{k+1}(\ell)=\mu_{k+1}(\ell)$, then because $u_{k}(\ell) \geq \mu_{k}(\ell)$, we have $u_{k}(\ell)-u_{k+1}(\ell) \geq \mu_{k}(\ell)-\mu_{k+1}(\ell)$.

2. If $u_{k+1}(\ell)>\mu_{k+1}(\ell)$, then

$$
\begin{aligned}
u_{k}(\ell)-u_{k+1}(\ell) & \geq F_{k}^{0}\left(\ell, \mathbf{u}_{k+1}\right)-F_{k+1}^{0}\left(\ell, \mathbf{u}_{k+2}\right) \\
& \geq F_{k+1}^{0}\left(\ell, \mathbf{u}_{k+1}-\mathbf{u}_{k+2}\right) \\
& \geq F_{k+1}^{0}\left(\ell, \boldsymbol{\mu}_{k+1}-\boldsymbol{\mu}_{k+2}\right) .
\end{aligned}
$$

Since $p_{t+1}^{0}(L+1 \mid \ell) \geq p_{t}^{0}(L+1 \mid \ell)$ for all $\ell \in \mathcal{L}$ and $t \in$ $T^{\prime} \backslash\{N-1\}$, by definition, $p_{k}^{0}\left(\ell^{\prime} \mid \ell\right) \geq p_{k+1}^{0}\left(\ell^{\prime} \mid \ell\right)$ for all $\ell^{\prime} \in \mathcal{L}^{\prime}$, and this yields the first inequality (7). Then, the second inequality is implied by the induction hypothesis. Since $B_{k+1}(\ell) \geq B_{k}(\ell)$, (7) implies $u_{k}(\ell)-u_{k+1}(\ell) \geq$ $\mu_{k}(\ell)-\mu_{k+1}(\ell)$.

Thus, $\mathbf{u}_{k}-\boldsymbol{\mu}_{k} \geq \mathbf{u}_{k+1}-\boldsymbol{\mu}_{k+1}$, and the result follows by induction on $t$.

If we consider $m$ continuous, $0 \leq m \leq 1$, because $\omega<1$, it can be easily shown that $\frac{\partial \pi_{t}^{C}(\ell, m)}{\partial m}$ and $\frac{\partial \pi_{t}^{S}(\ell, m)}{\partial m}$ are both nonpositive for all $\ell \in \mathcal{L}^{\prime}$. Then, by definition, it is easy to see that $p_{t}^{0}\left(\ell^{\prime} \mid \ell\right) \leq p_{t}^{1}\left(\ell^{\prime} \mid \ell\right)$ for all $\ell, \ell^{\prime} \in \mathcal{L}^{\prime}$ and $t \in T^{\prime}$. Also, given two patients with quality-adjustment factors ${ }^{1} \sigma \leq{ }^{2} \sigma$, if ${ }^{1} p_{t}^{1}\left(\ell^{\prime} \mid \ell\right) \geq{ }^{2} p_{t}^{1}\left(\ell^{\prime} \mid \ell\right)$ for $\ell, \ell^{\prime} \in \mathcal{L}^{\prime}$ and $t \in T^{\prime}$, then it can be shown that ${ }^{1} \mu_{t} \geq{ }^{2} \mu_{t}$ for all $t \in T$.

Proof. of Proposition 5: First, we will establish an auxiliary result. Given two patients with ${ }^{1} \sigma \leq{ }^{2} \sigma$, if

$$
\begin{aligned}
{ }^{1} p_{t}^{0}\left(\ell^{\prime} \mid \ell\right) & \leq{ }^{2} p_{t}^{0}\left(\ell^{\prime} \mid \ell\right), \text { and }{ }^{1} p_{t}^{1}\left(\ell^{\prime} \mid \ell\right) \\
& \geq{ }^{2} p_{t}^{1}\left(\ell^{\prime} \mid \ell\right) \text { for all } \ell, \ell^{\prime} \in \mathcal{L}^{\prime} \text { and } t \in T^{\prime},
\end{aligned}
$$

then for any $\ell \in \mathcal{L}^{\prime}$ and $t \in T,{ }^{2} a_{t}(\ell)=I$ implies ${ }^{1} a_{t}(\ell)=I$. To prove the claim, it is sufficient to show that ${ }^{1} \mathbf{u}_{t}-{ }^{1} \boldsymbol{\mu}_{t} \leq$ ${ }^{2} \mathbf{u}_{t}-{ }^{2} \boldsymbol{\mu}_{t}$ for all $t \in T$. To establish this result for $t=N$, we will proceed by induction on the iterates of the value iteration algorithm. We will apply the algorithm to ${ }^{i} \mathbf{u}_{N}$ simultaneously for both $i=1,2$. By the convergence of the algorithm it is sufficient to show that ${ }^{1} \mathbf{u}_{N}^{k}-{ }^{1} \boldsymbol{\mu}_{N} \leq$ ${ }^{2} \mathbf{u}_{N}^{k}-{ }^{2} \boldsymbol{\mu}_{N}$ for all $k \geq 0$. Initialize ${ }^{i} \mathbf{u}_{N}^{0}={ }^{i} \boldsymbol{\mu}_{N}$ for $i=1,2$ and for some $n \geq 0$, suppose ${ }^{1} \mathbf{u}_{N}^{n}-{ }^{1} \boldsymbol{\mu}_{N} \leq{ }^{2} \mathbf{u}_{N}^{n}-{ }^{2} \boldsymbol{\mu}_{N}$. Now, for an arbitrary $\ell \in \mathcal{L}^{\prime}$ consider the possible cases for ${ }^{2} u_{N}^{n+1}(\ell)-{ }^{1} u_{N}^{n+1}(\ell)$.

1. If ${ }^{1} u_{N}^{n+1}(\ell)={ }^{1} \mu_{N}(\ell)$, then because ${ }^{2} u_{N}^{n+1}(\ell) \geq{ }^{2} \mu_{N}(\ell)$, we have ${ }^{2} u_{N}^{n+1}(\ell)-{ }^{1} u_{N}^{n+1}(\ell) \geq{ }^{2} \mu_{N}(\ell)-{ }^{1} \mu_{N}(\ell)$.

2. If ${ }^{1} u_{N}^{n+1}(\ell)>{ }^{1} \mu_{N}(\ell)$, then

$$
\begin{aligned}
& { }^{2} u_{N}^{n+1}(\ell)-{ }^{1} u_{N}^{n+1}(\ell) \geq{ }^{2} F_{N-1}^{0}\left(\ell,{ }^{2} \mathbf{u}_{N}^{n}\right)-{ }^{1} F_{N-1}^{0}\left(\ell,{ }^{1} \mathbf{u}_{N}^{n}\right) \\
& \quad={ }^{2} F_{N-1}^{0}\left(\ell,{ }^{2} \mathbf{u}_{N}^{n}-{ }^{2} \boldsymbol{\mu}_{N}\right)+{ }^{2} F_{N-1}^{0}\left(\ell,{ }^{2} \boldsymbol{\mu}_{N}\right) \\
& \quad-{ }^{1} F_{N-1}^{0}\left(\ell,{ }^{1} \mathbf{u}_{N}^{n}-{ }^{1} \boldsymbol{\mu}_{N}\right)-{ }^{1} F_{N-1}^{0}\left(\ell,{ }^{1} \boldsymbol{\mu}_{N}\right) \\
& \geq{ }^{2} F_{N-1}^{0}\left(\ell,{ }^{1} \mathbf{u}_{N}^{n}-{ }^{1} \boldsymbol{\mu}_{N}\right)-{ }^{1} F_{N-1}^{0}\left(\ell,{ }^{1} \mathbf{u}_{N}^{n}-{ }^{1} \boldsymbol{\mu}_{N}\right) \\
& \quad+{ }^{2} F_{N-1}^{0}\left(\ell,{ }^{2} \boldsymbol{\mu}_{N}\right)-{ }^{1} F_{N-1}^{0}\left(\ell,{ }^{1} \boldsymbol{\mu}_{N}\right)
\end{aligned}
$$




$$
\begin{aligned}
& \geq{ }^{2} F_{N-1}^{0}\left(\ell,{ }^{2} \boldsymbol{\mu}_{N}\right)-{ }^{1} F_{N-1}^{0}\left(\ell,{ }^{1} \boldsymbol{\mu}_{N}\right) \\
& \geq{ }^{2} F_{N-1}^{1}\left(\ell,{ }^{2} \boldsymbol{\mu}_{N}\right)-{ }^{1} F_{N-1}^{1}\left(\ell,{ }^{1} \boldsymbol{\mu}_{N}\right)+\tau\left({ }^{1} \sigma-{ }^{2} \sigma\right) \\
& ={ }^{2} \mu_{N}(\ell)-{ }^{1} \mu_{N}(\ell),
\end{aligned}
$$

where (9) is implied by the fact that ${ }^{2} u_{N}^{n+1}(\ell) \geq r^{0}(\ell)+$ ${ }^{2} F_{N-1}^{0}\left(\ell,{ }^{2} \mathbf{u}_{N}^{n}\right)$ and (10) is implied by the induction hypothesis. Then, since ${ }^{2} p_{N-1}^{0}\left(\ell^{\prime} \mid \ell\right) \geq{ }^{1} p_{N-1}^{0}\left(\ell^{\prime} \mid \ell\right)$ and ${ }^{1} \mathbf{u}_{N}^{n} \geq{ }^{1} \boldsymbol{\mu}_{N}$ and ${ }^{1} u_{N}^{n}(L+1)={ }^{1} \mu_{N}(L+1)=0,(11)$ is implied by ${ }^{2} F_{N-1}^{0}\left(\ell,{ }^{1} \mathbf{u}_{N}^{n}-{ }^{1} \boldsymbol{\mu}_{N}\right) \geq{ }^{1} F_{N-1}^{0}\left(\ell,{ }^{1} \mathbf{u}_{N}^{n}-\right.$ $\left.{ }^{1} \boldsymbol{\mu}_{N}\right)$. Finally, we show the last inequality in (12) as follows: By (8), ${ }^{1} p_{N-1}^{1}\left(\ell^{\prime} \mid \ell\right)-{ }^{1} p_{N-1}^{0}\left(\ell^{\prime} \mid \ell\right) \geq$ ${ }^{2} p_{N-1}^{1}\left(\ell^{\prime} \mid \ell\right)-{ }^{2} p_{N-1}^{0}\left(\ell^{\prime} \mid \ell\right)$ for all $\ell, \ell^{\prime} \in \mathcal{L}^{\prime}$. Also, because ${ }^{1} \sigma \leq{ }^{2} \sigma$ and (8) holds, ${ }^{1} \mu_{N} \geq{ }^{2} \mu_{N}$. Because ${ }^{i} \mu_{N-1}(L+1)=0$ for both $i=1,2,{ }^{1} F_{N-1}^{1}\left(\ell,{ }^{1} \mu_{N}\right)-$ ${ }^{1} F_{N-1}^{0}\left(\ell,{ }^{1} \boldsymbol{\mu}_{N}\right) \geq{ }^{2} F_{N-1}^{1}\left(\ell,{ }^{2} \boldsymbol{\mu}_{N}\right)-{ }^{2} F_{N-1}^{0}\left(\ell,{ }^{2} \boldsymbol{\mu}_{N}\right)$. By the fact that ${ }^{1} \sigma \leq{ }^{2} \sigma$, this yields the last inequality in (12).

Thus, ${ }^{1} \mathbf{u}_{N}^{n+1}-{ }^{1} \boldsymbol{\mu}_{N} \leq{ }^{2} \mathbf{u}_{N}^{n+1}-{ }^{2} \boldsymbol{\mu}_{N}$, and the result for $t=N$ follows by induction. The rest of the proof is similar by backwards induction on $t \in T$, and is omitted.

Now, we will treat each "or" case separately, and omit the proof of "and" case since it is similar. If the patients are identical except ${ }^{1} \sigma \leq{ }^{2} \sigma$, then the result directly follows from the auxiliary result that has been shown above. Otherwise, if the patients are identical except ${ }^{1} \omega \geq$ ${ }^{2} \omega$, by (1a)-(2b), ${ }^{1} \pi_{t}^{\nabla}(\ell, 0)={ }^{2} \pi_{t}^{\nabla}(\ell, 0)$ and ${ }^{1} \pi_{t}^{\nabla}(\ell, 1) \leq$ ${ }^{2} \pi_{t}^{\nabla}(\ell, 1)$ for all $\ell \in \mathcal{L}^{\prime}$ and $t \in T^{\prime}$. Then, since ${ }^{1} q\left(\ell^{\prime} \mid \ell\right)=$ ${ }^{2} q\left(\ell^{\prime} \mid \ell\right)$ for all $\ell, \ell^{\prime} \in \mathcal{L}^{\prime}$ and ${ }^{1} d_{t}={ }^{2} d_{t}$ for all $t \in T^{\prime}$, by definition, we have ${ }^{1} p_{t}^{1}\left(\ell^{\prime} \mid \ell\right) \geq{ }^{2} p_{t}^{1}\left(\ell^{\prime} \mid \ell\right)$ and ${ }^{1} p_{t}^{0}\left(\ell^{\prime} \mid \ell\right)=$ ${ }^{2} p_{t}^{0}\left(\ell^{\prime} \mid \ell\right)$ for all $\ell, \ell^{\prime} \in \mathcal{L}^{\prime}$ and $t \in T^{\prime}$, and the result follows from the auxiliary result that has been shown above.

Proof. of Proposition 6: For brevity, we will establish the result only for $t=N$. Let ${ }^{i, j} \mu_{N}(\ell)$ denote the expected post-treatment reward of patient $i, i=1,2$, in state $\ell \in \mathcal{L}$ at epoch $t=N$ when her quality-adjustment factor is $\sigma_{j}, j=1,2$. First, we will show that ${ }^{1,2} \boldsymbol{\mu}_{N}-{ }^{1,1} \boldsymbol{\mu}_{N} \geq$ ${ }^{2,2} \boldsymbol{\mu}_{N}-{ }^{2,1} \boldsymbol{\mu}_{N}$. We will proceed by induction on the iterates of the value iteration algorithm. We will apply the algorithm to ${ }^{1, j} \boldsymbol{\mu}_{N}$ for both $j=1,2$. Initialize ${ }^{1, j} \boldsymbol{\mu}_{N}^{0}=$ ${ }^{2, j} \boldsymbol{\mu}_{N}$ for $j=1,2$ so that ${ }^{1,2} \boldsymbol{\mu}_{N}^{0}-{ }^{1,1} \boldsymbol{\mu}_{N}^{0} \geq{ }^{2,2} \boldsymbol{\mu}_{N}-{ }^{2,1} \boldsymbol{\mu}_{N}$. As the induction hypothesis, for some $n \geq 0$, suppose ${ }^{1,2} \boldsymbol{\mu}_{N}^{n}-{ }^{1,1} \boldsymbol{\mu}_{N}^{n} \geq{ }^{2,2} \boldsymbol{\mu}_{N}-{ }^{2,1} \boldsymbol{\mu}_{N}$. Then, for an arbitrary $\ell \in \mathcal{L}^{\prime}$,

$$
\begin{aligned}
& { }^{1,2} \mu_{N}^{n+1}(\ell)-{ }^{1,1} \mu_{N}^{n+1}(\ell)-\left[{ }^{2,2} \mu_{N}(\ell)-{ }^{2,1} \mu_{N}(\ell)\right] \\
& \quad={ }^{1} F_{N-1}^{1}\left(\ell,{ }^{1,2} \boldsymbol{\mu}_{N}^{n}-{ }^{1,1} \boldsymbol{\mu}_{N}^{n}\right)-{ }^{2} F_{N-1}^{1}\left(\ell,{ }^{2,2} \boldsymbol{\mu}_{N}-{ }^{2,1} \boldsymbol{\mu}_{N}\right) \\
& \geq{ }^{1} F_{N-1}^{1}\left(\ell,{ }^{1,2} \boldsymbol{\mu}_{N}^{n}-{ }^{1,1} \boldsymbol{\mu}_{N}^{n}-{ }^{2,2} \boldsymbol{\mu}_{N}+{ }^{2,1} \boldsymbol{\mu}_{N}\right) \geq 0 .
\end{aligned}
$$

Because the patients are identical except ${ }^{1} \omega \geq{ }^{2} \omega$ :

$$
\begin{aligned}
{ }^{1} p_{N-1}^{1}\left(\ell^{\prime} \mid \ell\right) & \geq{ }^{2} p_{N-1}^{1}\left(\ell^{\prime} \mid \ell\right) \text { and }{ }^{1} p_{N-1}^{0}\left(\ell^{\prime} \mid \ell\right) \\
& ={ }^{2} p_{N-1}^{0}\left(\ell^{\prime} \mid \ell\right) \text { for all } \ell, \ell^{\prime} \in \mathcal{L}^{\prime} .
\end{aligned}
$$

Now, because $\sigma_{1} \geq \sigma_{2}, \quad{ }^{2,2} \boldsymbol{\mu}_{N} \geq{ }^{2,1} \boldsymbol{\mu}_{N}$. Because ${ }^{2,2} \mu_{N}(L+1)={ }^{2,1} \mu_{N}(L+1)=0$, by (14) this implies ${ }^{1} F_{N-1}^{1}\left(\ell,{ }^{2,2} \boldsymbol{\mu}_{N}-{ }^{2,1} \boldsymbol{\mu}_{N}\right) \geq{ }^{2} F_{N-1}^{1}\left(\ell,{ }^{2,2} \boldsymbol{\mu}_{N}-{ }^{2,1} \boldsymbol{\mu}_{N}\right)$, implying the first inequality in (13). The second inequality in (13) directly follows from the induction hypothesis. Thus, ${ }^{1,2} \boldsymbol{\mu}_{N}^{n+1}-{ }^{1,1} \boldsymbol{\mu}_{N}^{n+1} \geq{ }^{2,2} \boldsymbol{\mu}_{N}-{ }^{2,1} \boldsymbol{\mu}_{N}$. Then, the convergence of the algorithm yields ${ }^{1,2} \boldsymbol{\mu}_{N}-{ }^{1,1} \boldsymbol{\mu}_{N} \geq{ }^{2,2} \boldsymbol{\mu}_{N}-{ }^{2,1} \boldsymbol{\mu}_{N}$. Next, for $t=N$, we will apply the algorithm simultaneously to ${ }^{i, j} \mathbf{u}_{N}$ for $i, j=1,2$ to show that ${ }^{1,2} \mathbf{u}_{N}-{ }^{1,1} \mathbf{u}_{N} \geq{ }^{2,2} \mathbf{u}_{N}-{ }^{2,1} \mathbf{u}_{N}$. We will proceed by induction on the iterates of the algorithm. Initialize ${ }^{i, j} \mathbf{u}_{N}^{0}={ }^{i, j} \boldsymbol{\mu}_{N}$ for $\ell \in \mathcal{L}$ and $i, j=1,2$. Because ${ }^{1,2} \boldsymbol{\mu}_{N}-{ }^{1,1} \boldsymbol{\mu}_{N} \geq{ }^{2,2} \boldsymbol{\mu}_{N}-{ }^{2,1} \boldsymbol{\mu}_{N}$, this implies ${ }^{1,2} \mathbf{u}_{N}^{0}-{ }^{1,1} \mathbf{u}_{N}^{0} \geq{ }^{2,2} \mathbf{u}_{N}^{0}-{ }^{2,1} \mathbf{u}_{N}^{0}$. For some $n \geq 0$, suppose ${ }^{1,2} \mathbf{u}_{N}^{n}-{ }^{1,1} \mathbf{u}_{N}^{n} \geq{ }^{2,2} \mathbf{u}_{N}^{n}-{ }^{2,1} \mathbf{u}_{N}^{n}$. We will make use of the following set of auxiliary results to establish ${ }^{1,2} \mathbf{u}_{N}^{n+1}-{ }^{1,1} \mathbf{u}_{N}^{n+1} \geq{ }^{2,2} \mathbf{u}_{N}^{n+1}-{ }^{2,1} \mathbf{u}_{N}^{n+1}$.

- Since $\sigma_{1} \geq \sigma_{2}$, by treating each patient as two identical patients with different quality-adjustment factors we have,

$$
{ }^{i, 1} \mathbf{u}_{N}^{n}-{ }^{i, 1} \boldsymbol{\mu}_{N} \geq{ }^{i, 2} \mathbf{u}_{N}^{n}-{ }^{i, 2} \boldsymbol{\mu}_{N} \text { for } i=1,2 .
$$

Similarly, since (14) is satisfied,

$$
{ }^{2, j} \mathbf{u}_{N}^{n}-{ }^{2, j} \boldsymbol{\mu}_{N} \geq{ }^{1, j} \mathbf{u}_{N}^{n}-{ }^{1, j} \boldsymbol{\mu}_{N} \text { for } j=1,2 .
$$

(The proofs of (15) and (16) are given in the proof of Proposition 5).

- Define ${ }^{i, j} U(\ell)=r^{0}(\ell)+{ }^{i} F_{N-1}^{0}\left(\ell,{ }^{i, j} \mathbf{u}_{N}^{n}\right)$ for $\ell \in \mathcal{L}$ and $i, j=1,2$. For an arbitrary $\ell \in \mathcal{L}$, consider ${ }^{1,2} U(\ell)-$ ${ }^{1,1} U(\ell)-\left[{ }^{2,2} U(\ell)-{ }^{2,1} U(\ell)\right]$.

$$
\begin{aligned}
& { }^{1,2} U(\ell)-{ }^{1,1} U(\ell)-\left[{ }^{2,2} U(\ell)-{ }^{2,1} U(\ell)\right]={ }^{1} F_{N-1}^{0} \\
& \quad \times\left(\ell,{ }^{1,2} \mathbf{u}_{N}^{n}-{ }^{1,1} \mathbf{u}_{N}^{n}\right)-{ }^{n} F_{N-1}^{0}\left(\ell,{ }^{2,2} \mathbf{u}_{N}^{n}-{ }^{2,1} \mathbf{u}_{N}^{n}\right) \geq 0,
\end{aligned}
$$

where the inequality is implied by the fact that ${ }^{2} p_{N-1}^{0}\left(\ell^{\prime} \mid \ell\right)={ }^{1} p_{N-1}^{0}\left(\ell^{\prime} \mid \ell\right)$ for all $\ell^{\prime} \in \mathcal{L}$ and the induction hypothesis. Thus, ${ }^{1,2} \mathbf{U}-{ }^{1,1} \mathbf{U} \geq{ }^{2,2} \mathbf{U}-{ }^{2,1} \mathbf{U}$.

- ${ }^{1,2} \boldsymbol{\mu}_{N}-{ }^{2,2} \boldsymbol{\mu}_{N} \geq{ }^{1,2} \mathbf{U}-{ }^{2,2} \mathbf{U}$. The proof of this fact is as follows. For fixed $\ell \in \mathcal{L}$ :

$$
\begin{aligned}
& { }^{1,2} \mu_{N}(\ell)-{ }^{2,2} \mu_{N}(\ell) \\
& \quad={ }^{1} F_{N-1}^{1}\left(\ell,{ }^{1,2} \boldsymbol{\mu}_{N}\right)-{ }^{2} F_{N-1}^{1}\left(\ell,{ }^{2,2} \boldsymbol{\mu}_{N}\right) \\
& \geq{ }^{1} F_{N-1}^{0}\left(\ell,{ }^{1,2} \boldsymbol{\mu}_{N}\right)-{ }^{2} F_{N-1}^{0}\left(\ell,{ }^{2,2} \boldsymbol{\mu}_{N}\right) \\
& \geq{ }^{1} F_{N-1}^{0}\left(\ell,{ }^{1,2} \mathbf{u}_{N}^{n}\right)-{ }^{2} F_{N-1}^{0}\left(\ell,{ }^{2,2} \mathbf{u}_{N}^{n}\right) \\
& ={ }^{1,2} U(\ell)-{ }^{2,2} U(\ell),
\end{aligned}
$$

Since ${ }^{1} p_{N-1}^{1}\left(\ell^{\prime} \mid \ell\right) \geq{ }^{2} p_{N-1}^{1}\left(\ell^{\prime} \mid \ell\right)$ for all $\ell^{\prime} \in \mathcal{L}$, it can be easily shown that ${ }^{1,2} \boldsymbol{\mu}_{N} \geq{ }^{2,2} \boldsymbol{\mu}_{N}$. Then, (by (14)), because ${ }^{1} p_{N-1}^{1}\left(\ell^{\prime} \mid \ell\right)-{ }^{1} p_{N-1}^{0}\left(\ell^{\prime} \mid \ell\right) \geq$ ${ }^{2} p_{N-1}^{1}\left(\ell^{\prime} \mid \ell\right)-{ }^{2} p_{N-1}^{0}\left(\ell^{\prime} \mid \ell\right) \geq 0$ for all $\ell, \ell^{\prime} \in$ $\mathcal{L}^{\prime}, \quad{ }^{1} F_{N-1}^{1}\left(\ell,{ }^{1,2} \mu_{N}\right)-{ }^{1} F_{N-1}^{0}\left(\ell,{ }^{1,2} \mu_{N}\right) \geq$ ${ }^{2} F_{N-1}^{1}\left(\ell,{ }^{2,2} \boldsymbol{\mu}_{N}\right)-{ }^{2} F_{N-1}^{0}\left(\ell,{ }^{2,2} \boldsymbol{\mu}_{N}\right)$, and this yields the inequality in (17). Then, the inequality in (18) is implied by ${ }^{1} F_{N-1}^{0}\left(\ell,{ }^{1,2} \boldsymbol{\mu}_{N}-{ }^{1,2} \mathbf{u}_{N}^{n}\right) \geq$ 
${ }^{2} F_{N-1}^{0}\left(\ell,{ }^{2,2} \boldsymbol{\mu}_{N}-{ }^{2,2} \mathbf{u}_{N}^{n}\right)$ which follows from (16) and the fact that ${ }^{1} p_{N-1}^{0}\left(\ell^{\prime} \mid \ell\right)={ }^{2} p_{N-1}^{0}\left(\ell^{\prime} \mid \ell\right)$ for all $\ell^{\prime} \in \mathcal{L}$.

Next, for an arbitrary $\ell \in \mathcal{L}^{\prime}$ consider the possible cases for ${ }^{1,2} u_{N}^{n+1}(\ell)-{ }^{2,2} u_{N}^{n+1}(\ell)$. Note that ${ }^{i, j} u_{N}^{n+1}(\ell)=$ $\max \left\{{ }^{i, j} U(\ell),{ }^{i, j} \mu_{N}(\ell)\right\}$ for $\ell \in \mathcal{L}$ and $i, j=1,2$.

1. If ${ }^{1,1} u_{N}^{n+1}(\ell)={ }^{1,1} \mu_{N}(\ell)$, then by $(15),{ }^{1,2} u_{N}^{n+1}(\ell) \leq$ ${ }^{1,2} \mu_{N}(\ell)$. By the definition of ${ }^{1,2} u_{N}^{n+1}(\ell)$, we also have ${ }^{1,2} u_{N}^{n+1}(\ell) \geq{ }^{1,2} \mu_{N}(\ell)$. These imply ${ }^{1,2} u_{N}^{n+1}(\ell)=$ $1,2 \mu_{N}(\ell)$. Then, by (16) we achieve

$$
\begin{aligned}
& { }^{1,2} u_{N}^{n+1}(\ell)-{ }^{1,1} u_{N}^{n+1}(\ell)-\left[{ }^{2,2} u_{N}^{n+1}(\ell)-{ }^{2,1} u_{N}^{n+1}(\ell)\right] \\
& ={ }^{1,2} \mu_{N}(\ell)-{ }^{1,1} \mu_{N}(\ell)-\left[{ }^{2,2} u_{N}^{n+1}(\ell)-{ }^{2,1} u_{N}^{n+1}(\ell)\right] \\
& \geq{ }^{1,2} \mu_{N}(\ell)-{ }^{1,1} \mu_{N}(\ell)-{ }^{2,2} \mu_{N}(\ell)+{ }^{2,1} \mu_{N}(\ell) \geq 0 .
\end{aligned}
$$

2. If ${ }^{1,1} u_{N}^{n+1}(\ell)={ }^{1,1} U(\ell)$, then by $(16),{ }^{2,1} u_{N}^{n+1}(\ell)=$ ${ }^{2,1} U(\ell)$. Now, consider the possible subcases:

(a) If ${ }^{2,2} u_{N}^{n+1}(\ell)={ }^{2,2} \mu_{N}(\ell)$, then by $(15),{ }^{1,2} u_{N}^{n+1}(\ell) \leq$ ${ }^{1,2} \mu_{N}(\ell)$. By the definition of ${ }^{1,2} u_{N}^{n+1}(\ell)$, we also have ${ }^{1,2} u_{N}^{n+1}(\ell) \geq{ }^{1,2} \mu_{N}(\ell)$. These imply ${ }^{1,2} u_{N}^{n+1}(\ell)=$ ${ }^{1,2} \mu_{N}(\ell)$. Then,

$$
\begin{aligned}
& { }^{1,2} u_{N}^{n+1}(\ell)-{ }^{1,1} u_{N}^{n+1}(\ell)-\left[{ }^{2,2} u_{N}^{n+1}(\ell)-{ }^{2,1} u_{N}^{n+1}(\ell)\right] \\
& ={ }^{1,2} \mu_{N}(\ell)-{ }^{1,1} U(\ell)-{ }^{2,2} \mu_{N}(\ell)+{ }^{2,1} U(\ell) \\
& \quad \geq{ }^{1,2} U(\ell)-{ }^{1,1} U(\ell)-{ }^{2,2} U(\ell)+{ }^{2,1} U(\ell) \geq 0 .
\end{aligned}
$$

(b) If ${ }^{2,2} u_{N}^{n+1}(\ell)={ }^{2,2} U_{N}(\ell)$, then because ${ }^{1,2} u_{N}^{n+1}(\ell) \geq$ ${ }^{1,2} U(\ell)$

$$
\begin{array}{r}
{ }^{1,2} u_{N}^{n+1}(\ell)-{ }^{1,1} u_{N}^{n+1}(\ell)-\left[{ }^{2,2} u_{N}^{n+1}(\ell)-{ }^{2,1} u_{N}^{n+1}(\ell)\right] \\
\geq{ }^{1,2} U(\ell)-{ }^{1,1} U(\ell)-\left[{ }^{2,2} U(\ell)-{ }^{2,1} U(\ell)\right] \geq 0 .
\end{array}
$$

Thus, ${ }^{1,2} \mathbf{u}_{N}^{n+1}-{ }^{1,1} \mathbf{u}_{N}^{n+1} \geq{ }^{2,2} \mathbf{u}_{N}^{n+1}-{ }^{2,1} \mathbf{u}_{N}^{n+1}$. Then, the convergence of the algorithm yields ${ }^{1,2} \mathbf{u}_{N}-{ }^{1,1} \mathbf{u}_{N} \geq$ ${ }^{2,2} \mathbf{u}_{N}-{ }^{2,1} \mathbf{u}_{N}$. The proof for the case $t<N$ is similar by backwards induction on $t \in T$.

\section{Appendix E: Measuring the Violations of the Assumptions}

In this section we evaluate the maximum violations of the assumptions and the conditions presented in Section 3. We let $a^{+}=\max \{a, 0\}$ for $a \in \mathbb{R}$ and quantify the magnitudes of the maximum violations of the assumptions by the metrics below.

- For $\mathbf{P}^{m}(t)$ being IFR, $\epsilon_{1}=\quad \max$

$$
\left[\sum_{\ell^{\prime}=1}^{k}\left[p_{t}^{m}\left(\ell^{\prime} \mid \ell+1\right)-p_{t}^{m}\left(\ell^{\prime} \mid \ell\right)\right]^{+}\right. \text {. }
$$

- For $B_{t}(\ell)$ being nondecreasing in

$$
\ell \in \mathcal{L}^{\prime}, \epsilon_{2}=\max _{\ell \in \mathcal{L}^{\prime} \backslash\{L\}, t \in T^{\prime}}\left[B_{t}(\ell)-B_{t}(\ell+1)\right]^{+} .
$$

- For the time-monotonicity of the terminal event probabilities, $\epsilon_{3}=\max _{\ell \in \mathcal{L}^{\prime}, m \in \mathcal{M}, t \in T^{\prime} \backslash\{N-1\}}$

$$
\left[p_{t}^{m}(L+1 \mid \ell)-p_{t+1}^{m}(L+1 \mid \ell)\right]^{+}(0.01) \text {. }
$$

- For $B_{t}(\ell)$ being nondecreasing in

$$
t \in T^{\prime}, \epsilon_{4}=\max _{\ell \in \mathcal{L}^{\prime}, t \in T^{\prime} \backslash\{N-1\}}\left[B_{t}(\ell)-B_{t+1}(\ell)\right]^{+} .
$$

For our data set, the maximum value of the metrics $\epsilon_{1}-$ $\epsilon_{3}$ are observed as follows: $\epsilon_{1}=0.0243, \epsilon_{2}=0.0245$ and $\epsilon_{3}=0.0171$. We also observe that $B_{t}(\ell)$ is nondecreasing in $t \in T^{\prime}$ for all $\ell \in \mathcal{L}$ in all of our instances; that is, $\epsilon_{4}=0$ across our experiments.

\section{References}

Denton, B. T., Kurt, M., Shah, N. D., Bryant, S. C., and Smith, S. A. (2009). Optimizing the start time of statin therapy for patients with diabetes. Medical Decision Making, 29(3), 351-368.

Gorman, C. A., Zimmerman, B. R., Smith, S. A., Dinneen, S. F., Knudsen, J. B., Holm, D., Jorgensen, B., Bjornsen, S., Planet, K., Hanson, P., and Rizza, R. A. ( 2000). DEMS - a second generation diabetes electronic management system. Computer Methods and Programs in Biomedicine, 62(2), 127-140.

Hebert, P. R., Gaziano, J. M., Chan, K. S., and Hennekens, C. H. (1997). Cholesterol lowering with statin drugs, risk of stroke, and total mortality - an overview of randomized trials. Journal of the American Medical Association, 278(4), 313-321.

Kothari, V., Stevens, R. J., Adler, A. I., Stratton, I. M., Manley, S. E., Neil, H. A., and Holman, R. R. (2002). UKPDS 60 - risk of stroke in Type 2 diabetes estimated by the UK Prospective Diabetes Study risk engine. Stroke, 33(7), 1776-1781,

Maron, D. J., Fazio, S., and Linton, M. F. (2000). Current perspectives on statins. Circulation, 101(2), 207-213.

National Center for Health Statistics (NCHS). (2009). Mortality data from the National Vital Statistics System, 2005. Information and data accessed on November 1, 2009, http://www.cdc.gov/nchs/deaths.htm.

National Cholesterol Education Program. (2001). Executive summary of the $3^{\text {rd }}$ report of the NCEP expert panel on detection, evaluation, and treatment of high blood cholesterol in adults (Adult Treatment Panel III). Journal of the American Medical Association, 285(19), 2486-2497.

Puterman, M. L. (1994). Markov Decision Processes: Discrete Stochastic Dynamic Programming. John Wiley \& Sons, New York.

Shechter, S. M. (2006). Optimal Scheduling of Highly Active Antiretroviral Therapies for HIV Patients. PhD thesis, University of Pittsburgh, USA.

Snow, V., Aronson, M. D., Hornbake, E. R., Mottur-Pilson, C., and Weiss, K. B. (2004). Lipid control in the management of Type 2 diabetes mellitus: A clinical practice guideline from the American College of Physicians. Annals of Internal Medicine, 140(8), 644-649.

Stevens, R. J., Kothari, V., Adler, A. I., Stratton, I. M., and Holman, R. R. (2001). The UKPDS risk engine: A model for the risk of coronary heart disease in Type 2 diabetes (UKPDS 56). Clinical Science, 101(6), 671-679.

Vijan, S. and Hayward, R. A. (2004). Pharmacologic lipid-lowering therapy in Type 2 diabetes mellitus: Background paper for the American College of Physicians. Annals of Internal Medicine, 140(8), 650-658.

Warnick, G. R., Knopp, R. H., Fitzpatrick, V., and Branson, L. (1990). Estimating low-density lipoprotein cholesterol by the Friedewald equation is adequate for classifying patients on the basis of nationally recommended cutpoints. Clinical Chemistry, 36(1), 15-19. 